University of New Orleans

ScholarWorks@UNO

1994

\title{
The physics of grain-grain collisions and gas-grain sputtering in interstellar shocks
}

\author{
A G. Tielens \\ C F. McKee \\ C G. Seab \\ University of New Orleans \\ D J. Hollenbach
}

Follow this and additional works at: https://scholarworks.uno.edu/phys_facpubs

Part of the Physics Commons

\section{Recommended Citation}

Astrophys. J. 431321 (1994)

This Article is brought to you for free and open access by the Department of Physics at ScholarWorks@UNO. It has been accepted for inclusion in Physics Faculty Publications by an authorized administrator of ScholarWorks@UNO.

For more information, please contact scholarworks@uno.edu. 


\title{
THE PHYSICS OF GRAIN-GRAIN COLLISIONS AND GAS-GRAIN SPUTTERING IN INTERSTELLAR SHOCKS
}

\author{
A. G. G. M. Tielens, ${ }^{1}$ C. F. McKee, ${ }^{2}$ C. G. Seab, ${ }^{3}$ And D. J. HollenbaCh ${ }^{1}$ \\ Received 1993 December 27; accepted 1994 February 11
}

\begin{abstract}
Grain-grain collisions and ion sputtering destroy dust grains in interstellar shocks. An analytical theory is developed for the propagation of shock waves in solids driven by grain-grain collisions, which compares very favorably with detailed numerical calculations. This theory is used to determine the fraction of a grain vaporized by a grain-grain collision. Our results predict much less vaporization of colliding grains in interstellar shocks than previous estimates. This theory can also be used to determine the fraction of a colliding grain that melts, shatters, or undergoes a phase transformation to a higher density phase. In particular, the latter two processes can be much more important in interstellar shocks than vaporization.

The sputtering of grains by impacting gas ions is reanalyzed based upon extensive laboratory studies and a theoretically derived "universal" sputtering relation. The analytical results are compared to available experimental studies of sputtering of graphite/amorphous carbon, $\mathrm{SiO}_{2}, \mathrm{SiC}, \mathrm{Fe}$, and $\mathrm{H}_{2} \mathrm{O}$. Sputtering yields for astrophysically relevant materials as a function of impact energy and ion mass are derived. These yields are also averaged over thermal impact spectrum and simple polynomial fits to the resulting yields as a function of temperature are presented. The derived sputtering yields are similar to those adopted in previous studies, except for graphite near threshold where the new yields are much larger due to a lower adopted binding energy. The ion bombardment will amorphitize the surface layers of interstellar grains. It will also convert graphite into hydrogenated amorphous carbon (HAC) to a depth of 10-20 $\AA$. It is suggested that these HAC surfaces are the carriers of the $3.4 \mu \mathrm{m}$ absorption feature in the interstellar medium.
\end{abstract}

Subject headings: dust, extinction - shock waves

\section{INTRODUCTION}

Interstellar dust is an important component of the interstellar medium (Allamandola \& Tielens 1989). Dust grains are the dominant opacity source for photons longward of the Lyman limit, and they therefore dominate the radiative balance of the interstellar medium. Photoelectrons from dust grains heat neutral atomic and molecular gas (Watson 1973; Tielens \& Hollenbach 1985). Collisions between dust and gas can also be an important energy source for the gas in dense molecular clouds. Furthermore, dust grains regulate the penetration of far-ultraviolet photons which can dissociate and ionize molecules. In that way, dust has an important influence on the composition and structure of molecular clouds and on the rate at which stars form in these clouds (McKee 1989a). Dust grains can also have a more direct contribution to the gas phase composition of molecular clouds since they provide surfaces for reaction. Unquestionably, the dominant interstellar molecule, $\mathrm{H}_{2}$, is formed by recombination of $\mathrm{H}$ atoms on grain surfaces and other molecules $\left(\mathrm{H}_{2} \mathrm{O}, \mathrm{NH}_{3}, \mathrm{H}_{2} \mathrm{CO}\right)$ may be formed that way as well (Hollenbach \& Salpeter 1971; Tielens \& Hagen 1982; Tielens \& Allamandola 1987).

Stardust is formed in the outflows from red giants, supergiants, novae, and supernovae and injected into the interstellar medium with the gas. This stardust consists of silicates and carbon soot (aromatic structures connected by aliphatic chains) with trace amounts of $\mathrm{SiC}$, aluminum oxides, and polycyclic aromatic hydrocarbon molecules (Gehrz 1989; Tielens

\footnotetext{
${ }^{1}$ NASA/Ames Research Center, Mail Stop 245-3, Moffett Field, CA 940351000.

${ }^{2}$ Astronomy Department and Physics Department, University of California, Berkeley, CA 94720.

${ }^{3}$ Physics Department, University of New Orleans, New Orleans, LA 70148.
}

1990). In the interstellar medium, the gas and dust cycles rapidly between the different phases (cloud and intercloud). Inside molecular clouds, accretion and reaction on grain surfaces will lead to the formation of an icy grain mantle consisting of simple molecules such as $\mathrm{H}_{2} \mathrm{O}, \mathrm{NH}_{3}, \mathrm{H}_{2} \mathrm{CO}$, and $\mathrm{CH}_{3} \mathrm{OH}$. FUV photolysis and warm-up of this icy mantle will lead to the formation of an organic residue mantle (e.g., Tielens \& Allamandola 1987). Prolonged exposure of this residue to FUV in the diffuse medium may even lead to more or less complete carbonization and the formation of amorphous aromatic carbon structure (Jenniskens et al. 1993). Dust grains are destroyed by strong shock waves driven by, for example, supernova explosions and estimated lifetimes for dust grains in the interstellar medium are only about $5 \times 10^{8} \mathrm{yr}$ (Seab 1987; McKee 1989b). In contrast, the time for stellar winds and supernovae to replenish the stardust in the interstellar medium is about $2.5 \times 10^{9} \mathrm{yr}$ (Jones \& Tielens 1994). Hence, the expected abundance of stardust in the interstellar medium is small. In contrast, observations of interstellar extinction as well as of the depletions of various rock-forming elements (notably, $\mathrm{Fe}, \mathrm{Si}, \mathrm{Ca}$, and $\mathrm{Al}$ ) indicate that the dust-to-gas ratio is much larger than these theoretical estimates for the (star)dust-to-gas ratio would indicate. Hence, a large fraction of the dust in the interstellar medium must have formed in situ by processes such as accretion and FUV photolysis.

This lifetime argument depends to a large extent on theoretical studies of the destruction by sputtering and grain-grain collisions in interstellar shock waves. Over the years, very detailed models of interstellar shock waves and their effects on dust grains have been developed (Barlow 1978a, b; Shull 1978; Draine \& Salpeter 1979b; Seab \& Shull 1983; McKee et al. 1987). We have started a program at NASA/Ames and the University of California at Berkeley to reexamine this question. 
Here, we examine in detail various physical processes that play a role in the destruction of dust grains in interstellar shocks. In $\S 2$, we study the propagation of strong shock waves in solids driven by high-velocity grain-grain collisions. The fraction of the colliding grains vaporized by such collisions is evaluated in $\S 3$ as a function of material parameters and collision velocity. In $\S 4$, we reexamine sputtering yields for impacts on various astrophysically relevant materials. Finally, our results are summarized in $\S 5$. The formalism described in this study has already been used to estimate the fraction of graphite/ amorphous carbon converted into diamonds by grain-grain collisions in interstellar shocks (Tielens et al. 1987). In a further study (Jones et al. 1993), we use these newly derived vaporization and sputtering yields to reevaluate the destruction of dust grains in interstellar shocks and the lifetime of interstellar grains. Finally, grain-grain collisions can lead to shattering of the colliding grains. The theoretical framework derived here for vaporization can also be applied to estimate the effects of shattering, and this will be discussed in an astrophysical context in Jones et al. (1994).

\section{DYNAMICS OF GRAIN-GRAIN COLLISIONS}

When two solid particles collide at high velocity, strong shock waves are driven into each particle, compressing them to high pressures. Upon release, the shocked material may pass from the solid phase into the vapor phase or liquid phase, or it may shatter. In this section we shall develop a simple physical model for the collision of a finite solid particle (the projectile) with a semi-infinite solid (the target) in order to estimate the volumes of vaporized and shattered material.

Two distinct stages may be discerned in the collision (Dienes $\&$ Walsh 1970). In the first stage, planar shocks are driven into both the target and the projectile at nearly constant velocity and pressure. This stage ends when the shock wave in the projectile has reached the back surface and is reflected as a rarefaction wave. The second, or late, stage commences when this rarefaction reaches the shock in the target, weakening it and altering it into a more or less hemispherical form. The rarefaction also induces a backsplash of ejected material, which can carry away several times the momentum of the projectile. Of course, the projectile never enters this second stage. The same general features are expected in the collision of two dust grains of disparate size; if the grains are comparable in size, the second stage will be absent.

\subsection{Shocks in Solids}

An excellent review of the physics of shocks in solids has been given by Zel'dovich \& Raizer (1966). Here we shall breifly summarize the pertinent results and cast them into a form suitable for our analysis.

The essential difference between shocks in solids and shocks in gases is that the interactions among the closely packed atoms in a solid lead to a finite sound speed at zero pressure. The specific energy $\epsilon$ of the solid has an elastic component $\epsilon_{c}$, which is due to the interactions and depends only on the density $\rho$, in addition to the thermal component; the pressure $\boldsymbol{P}$ has corresponding elastic and thermal components. At zero temperature the thermal contributions vanish, and, in equilibrium, the repulsive and attractive forces between the atoms balance so that the elastic components vanish as well. If the density is raised above the equilibrium value $\rho_{0}$, then $\epsilon_{c}$ and $P_{c}$ rise rapidly, approaching the values appropriate to a degenerate electron gas. On the other hand, if the density is lowered below $\rho_{0}$, the attractive forces dominate so that $\epsilon_{c}$ increases, approaching the binding energy, whereas $P_{c}$ is negative. Fluctuations in $P_{c}$ about zero produce sound waves with a velocity of several $\mathrm{km} \mathrm{s}^{-1}$.

A shock in a gas is said to be strong if it is highly supersonic, or, equivalently, if the pressure behind the shock greatly exceeds that in front. In a solid these two criteria are no longer equivalent. We shall generally be interested in shocks in solids in which the initial pressure is indeed negligible, but which are only mildly supersonic. Since the shock is not highly supersonic, the compression is less than the factor 4 found in a strong shock in a monatomic gas.

The jump conditions, or Rankine-Hugoniot conditions, express conservation of mass, momentum, and energy across the shock front. For a shock in a solid with negligible initial internal energy and pressure, these jump conditions are identical to those for a strong shock in a gas (McKee \& Hollenbach 1980):

$$
\begin{gathered}
\rho_{1}\left(v_{s}-v_{1}\right)=\rho_{0} v_{s}, \\
P_{1}+\rho_{1}\left(v_{s}-v_{1}\right)^{2}=\rho_{0} v_{s}^{2}, \\
\epsilon_{1}+\frac{P_{1}}{\rho_{1}}+\frac{1}{2}\left(v_{s}-v_{1}\right)^{2}=\frac{1}{2} v_{s}^{2},
\end{gathered}
$$

where quantities just behind the shock are denoted by the subscript 1 , and where velocities are evaluated in the frame of the unshocked matter; $v_{s}$ is the shock velocity and $v_{1}$ the velocity of the shocked matter. With the aid of equation (2.1), the momentum jump condition can be rewritten as

$$
P_{1}=\rho_{0} v_{1} v_{s} \text {. }
$$

Similarly, the energy jump condition can be simplified to

$$
\epsilon_{1}=\frac{1}{2} v_{1}^{2}
$$

which is a direct statement of energy conservation in the frame of the shocked matter.

As remarked above, the difference between shocks in solids and shocks in gases is in the equation of state. For an ideal gas the equation of state is $P=(\gamma-1) \rho \epsilon$, where $\gamma$ is the ratio of specific heats. For a solid, the equation of state can be quite complicated; nonetheless, we may define an effective shock $\gamma$ by

$$
P_{1} \equiv(\gamma-1) \rho_{1} \epsilon_{1}
$$

For an ideal gas, $\gamma$ is constant, but in a solid $\gamma$ depends on the shock velocity. Since the jump conditions are identical to those for a strong shock in a gas, it follows that

$$
\frac{v_{1}}{v_{s}}=\frac{P_{1}}{\rho_{0} v_{s}^{2}}=\frac{2}{\gamma+1} .
$$

The strong repulsive forces in the shocked solid make it stiffer than a gas, so that $\gamma$ generally exceeds $5 / 3$.

Experimentally, it has been found that for a non-porous medium there is a simple linear relationship between the shock velocity and the velocity of the shocked matter over a wide range of shock pressures (McQueen et al. 1970):

$$
v_{s}=c_{0}+s v_{1} \text {. }
$$

The constant $c_{0}$ is approximately equal to the sound speed. Experimental values of $c_{0}$ and $s$ for several astrophysically relevant materials are given in Table 1. Small nonlinearities, 
TABLE 1

VAPORIzATION UPON Grain-Grain Collision

\begin{tabular}{|c|c|c|c|c|c|c|}
\hline \multirow[b]{2}{*}{ MATERIAL } & \multicolumn{3}{|c|}{ HUGONIOT $^{\mathbf{a}}$} & \multicolumn{3}{|c|}{ VAPORIZATION THRESHOLD $^{b}$} \\
\hline & $\begin{array}{c}\rho_{0} \\
\left(\mathrm{~g} \mathrm{~cm}^{-3}\right)\end{array}$ & $\left(\mathrm{km} \mathrm{s}^{c_{0}}\right)$ & $s$ & $\begin{array}{c}\epsilon_{v} \\
\left(10^{11} \text { ergs }^{-1}\right)\end{array}$ & $\begin{array}{c}P_{t} \\
\left(10^{12}{\left.\text { dyn } \mathrm{cm}^{-2}\right)}^{-2}\right.\end{array}$ & $\left(\begin{array}{c}v_{t} \\
\left(\mathrm{~km} \mathrm{~s}^{-1}\right)\end{array}\right.$ \\
\hline Silicate $\ldots \ldots \ldots \ldots \ldots \ldots \ldots \ldots \ldots$ & 3.3 & 5.0 & 1.23 & 4.8 & 5.4 & 19 \\
\hline Graphite/amorphous carbon ..... & 2.2 & 1.8 & 1.9 & 6.4 & 5.8 & 23 \\
\hline 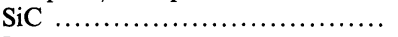 & 3.1 & 7.7 & 1.1 & 8.1 & 8.6 & 25 \\
\hline Ice $\ldots \ldots \ldots \ldots \ldots \ldots \ldots \ldots \ldots \ldots \ldots \ldots \ldots$ & 1.0 & 2.0 & 1.4 & 0.54 & 0.092 & 6.5 \\
\hline Iron $\ldots \ldots \ldots \ldots \ldots \ldots \ldots \ldots \ldots \ldots \ldots$ & 7.9 & 4.1 & 1.5 & 1.4 & 5.2 & 11 \\
\hline Gabbroic Anorthosite ............ & 2.9 & 4.6 & 1.2 & 3.4 & 3.6 & 16.5 \\
\hline
\end{tabular}

${ }^{\text {a }}$ Hugoniot data taken from McQueen et al. 1970 and Marsh 1980.

b Vaporization threshold correspond to $\epsilon_{v}=2 \epsilon_{b}$, with $\epsilon_{b}$ the binding energy; $v_{t}=2\left(2 \epsilon_{v}\right)^{1 / 2}$ for like materials impacting; $P_{t}$ has been calculated from the shock Hugoniot data.

which are due to the effect of phase changes, have been neglected by fitting a straight line through the high velocity portion of the data. The shock $\gamma$ in equations (2.6) and (2.7) is related to $c_{0}$ and $s$ by

$$
\gamma+1=2\left(s+\frac{c_{0}}{v_{1}}\right)=2\left(s+\mathscr{M}_{1}^{-1}\right),
$$

where

$$
\mathscr{M}_{1} \equiv v_{1} / c_{0}
$$

is the Mach number of the shocked material. Equation (2.9) emphasizes that $\gamma$ is not constant. At highly supersonic velocities $\left(v_{s} \gg c_{0}\right)$. which are beyond the range of existing experiments, $\gamma$ approaches $5 / 3$ and $s$ approaches $4 / 3$, comparable to the values in Table 1. The relative constancy of $s$ for the entire range of shock velocities of interest makes equation (2.8) quite useful.

\subsection{Collision Dynamics: First Stage}

In the first stage of a grain-grain collision, much of the relative kinetic energy of the two grains is thermalized. This stage is analogous to the ejecta-dominated stage in the evolution of a supernova remnant, in which the pressure of the swept-up interstellar medium drives a reverse shock into the supernova ejecta.

A simple one-dimensional model of the collision of two grains is shown in Figure 1. Shocks are driven into each grain; the grain which is crossed first is labeled the projectile. The projectile strikes the target at a relative velocity $v_{r}$. In the frame of the target, the velocities of the shocked target and shocked projectile are $v_{1}$ and $v_{1 P}^{\prime}$, respectively; these are equal since the material sandwiched between the two shocks has a single velocity. Now, the shock jump conditions for the projectile are given in the projectile frame, in which the velocity of the shocked projectile material (defined to be positive) is $v_{1 P}=$ $v_{r}-v_{1 P}^{\prime}=v_{r}-v_{1}$, so that

$$
v_{r}=v_{1}+v_{1 P} .
$$

The destructiveness of a grain-grain collision increases as $v_{r}^{2}$, so we focus on high velocity impacts in which the experimental relation (2.8) reduces to $v_{s} \simeq s v_{1}$. The pressure of the shocked projectile is identical to that of the shocked target, so the jump condition (2.4) yields

$$
\rho_{0} s v_{1}^{2}=\rho_{0 P} S_{P} v_{1 P}^{2}
$$

where $\rho_{0}$ is the preshock density of the target and $\rho_{O P}$ is the preshock density of the projectile. Defining

$$
\mathscr{R} \equiv\left(\frac{s \rho_{0}}{s_{P} \rho_{0 P}}\right)^{1 / 2}
$$

we obtain the results

$$
\begin{aligned}
v_{1} & =v_{r} /(1+\mathscr{R}), \\
v_{1 P} & =\mathscr{R} v_{r} /(1+\mathscr{R}) .
\end{aligned}
$$

For the collision of two identical substances $(\mathscr{R}=1)$, this reduces to $v_{1}=v_{1 P}=v_{r} / 2$, a result which is valid for all velocities by symmetry. The condition that equation (2.14) for $v_{1}$ be a good approximation is that $c_{0}$ be small compared to $s v_{1}$, or, since $s \sim 1$, that $\mathscr{M}_{r} \equiv v_{r} / c_{0} \gg(1+\mathscr{R})$; the fractional error in this equation is $\lesssim \frac{1}{2}\left(\mathscr{R} / \mathscr{M}_{r}\right)$. The initial pressure of the shocked target material can be inferred from equations (2.7) and (2.14):

$$
\begin{aligned}
\frac{P_{1 i}}{\rho_{0} c_{0}^{2}} & =\left(\frac{\gamma+1}{2}\right) \frac{\mathscr{M}_{r}^{2}}{(1+\mathscr{R})^{2}}, \\
& =\left(s+\frac{1+\mathscr{R}}{\mathscr{M}_{r}}\right) \frac{\mathscr{M}_{r}^{2}}{(1+\mathscr{R})^{2}} .
\end{aligned}
$$

The energy $E_{i}$ of the impact which is transmitted to the target is less than the relative energy $E_{0}=\frac{1}{2} M_{P} v_{r}^{2}$, where $M_{P}$ is the projectile mass, because of the energy carried away by the shocked projectile. If we equate this lost energy to the thermal

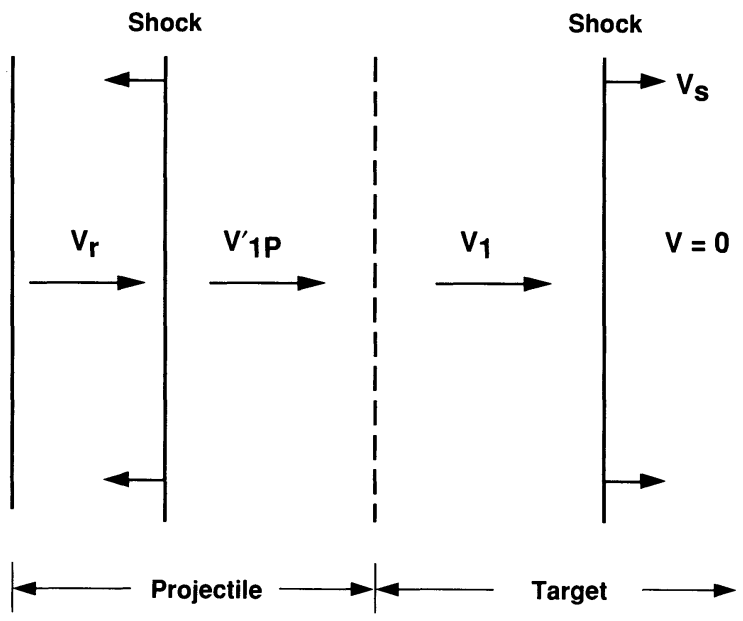

FIG. 1.-Schematic of the velocities involved in solid state shocks 
energy of the shocked projectile, $\frac{1}{2} M_{P} v_{1 P}^{2}$, we find

$$
E_{i}=\frac{1}{2} M_{P} v_{r}^{2} \frac{(1+2 \mathscr{R})}{(1+\mathscr{R})^{2}} .
$$

For identical materials, this reduces to $E_{i}=\frac{3}{4} E_{0}$. For a relatively dense projectile $(\mathscr{R} \ll 1)$, all the energy is transmitted to the target, whereas for a low-density projectile $(\mathscr{R} \gg 1)$, only a small fraction is $\left(E_{i} \rightarrow 2 E_{0} / \mathscr{R}\right)$.

\subsection{Collision Dynamics: Late Stage-The Impact Blast Wave}

When the shock driven into the target by the impact of the projectile has swept up much more mass than in the projectile, the flow becomes a blast wave which is independent of the details of the projectile. If, in addition, the shock velocity is large compared to the sound speed, then the flow becomes self-similar and the radius increases as a power of the time, $R \propto t^{\eta}$ (Zel'dovich \& Raizer 1966; Rae 1970). In contrast to the spherically symmetric case, the hot shocked material in the interior is exposed to the vacuum beyond the impact surface and flows outward, draining energy and imparting momentum to the blast wave. Hence, $\eta$ lies between the values $\frac{2}{5}$ for an energy conserving blast wave and $\frac{1}{4}$ for a momentum conserving one. For a self-similar blast wave with energy loss, the energy scales as a power of the radius, $E \propto R^{-k_{E}}$. Dimensional analysis then gives

$$
\eta=\frac{2}{5+k_{E}}
$$

(Ostriker \& McKee 1988). The decrease in energy $E$ and increase in momentum $p$ leave the quantity $M v_{s}^{3 \alpha} \propto$ $E^{3 \alpha-1} p^{2-3 \alpha}$ constant, where $M$ is the swept-up mass and $\alpha=$ $\eta /(1-\eta)=2 /\left(3+k_{E}\right)$. Thus the dynamics of an impact blast wave is determined by both the energy and the momentum of the collision. The value of $\alpha$ depends on $\gamma$ and is constrained to lie between $\frac{1}{3}$ and $\frac{2}{3}$. The numerical studies discussed in the Appendix give $\alpha \simeq 0.58-0.60$, consistent with the analytic model we develop there. We adopt

$$
k_{E}=\frac{3}{8}, \quad \eta=0.372, \quad \alpha=0.593 \text {. }
$$

Since $\alpha$ is not determined by dimensional analysis, this is a self-similar flow of the second type (Barenblatt \& Zel'dovich 1972).

When the shock slows, so that the Mach number $\mathscr{M}_{1}$ is no longer very large, the flow ceases to be self-similar and $M v_{s}^{3 \alpha}$ is no longer constant. However, since in a solid the pressure ahead of the shock remains zero, no new length scale is introduced, and the flow continues to be characterized by the initial value of $M v_{s}^{3 \alpha}$, which is proportional to $M_{P} v_{r}^{3 \alpha}(\operatorname{Rae} 1970)$. Two collisions with the same value of $M_{P} v_{r}^{3 \alpha}$ will produce identical craters (Dienes \& Walsh 1970).

The dynamics of energy conserving blast waves in solids has been discussed by Rao (1985), using techniques described by Rae (1970). Here we shall generalize this treatment to allow for the energy loss inherent in impact blast waves. If the blast wave is self-similar, then the energy is proportional to $M v_{s}^{2}$. One can then write $E=\sigma M v_{s}^{2}$, where $\sigma$ is a numerical constant that is typically of order unity (Ostriker \& McKee 1988); $\sigma$ is proportional to the quantity $I$ used by Rae and by Rao. Equivalently, in terms of $M v_{1}^{2}$, one has

$$
E=\sigma_{1} M v_{1}^{2}=\sigma_{1} M c_{0}^{2} \mathscr{M}_{1}^{2},
$$

where $\sigma_{1}=\sigma_{1}\left(\gamma, k_{E}\right)=(\gamma+1)^{2} \sigma / 4$ and $c_{0}$ is a constant of order the sound speed; for $c, s$ materials, which obey equation (2.8), $c_{0}$ is the constant entering that equation. For a self-similar, energy-losing blast wave, the energy obeys $E \propto R^{-k_{E}}$ (see eq. [2.18]), or equivalently,

$$
E=E_{i}\left(\frac{M}{M_{i}}\right)^{-k_{E} / 3},
$$

where $M_{i}$ is the shocked mass at the time that $E=E_{i}$. Evaluating equation (2.20) at $M=M_{i}$ implies

$$
M_{i}=\frac{E_{i}}{\sigma_{1 i} \mathscr{M}_{1 i}^{2} c_{0}^{2}}
$$

where $\sigma_{1 i}$ is the value of $\sigma_{1}$ at $\mathscr{M}=\mathscr{M}_{1 i}$. Combining equations (2.20) and (2.21), we then obtain the general relation between the shocked mass $M$ and the Mach number $\mathscr{M}_{1}$ :

$$
M=\frac{E_{i} / c_{0}^{2}}{\left(\sigma_{1 i} \mathscr{M}_{1 i}^{2}\right)^{k_{E} /\left(3+k_{E}\right)}\left(\sigma_{1} \mathscr{M}_{1}^{2}\right)^{3 /\left(3+k_{E}\right)}} .
$$

This reduces to Rao's (1985) result for $k_{E}=0$. For an impact blast wave, we identify $E_{i}$ with the energy transmitted to the target, equation (2.17), and replace $\mathscr{M}_{1 i}$ by $\mathscr{M}_{r} /(1+\mathscr{R})$ from equation (2.14), so that

$$
\frac{M}{M_{P}}=\frac{(1+2 \mathscr{R})}{2(1+\mathscr{R})^{6 /\left(3+k_{E}\right)}} \frac{1}{\sigma_{1 i}^{k_{E} /\left(3+k_{E}\right)}}\left(\frac{\mathscr{M}_{r}^{2}}{\sigma_{1} \mathscr{M}_{1}^{2}}\right)^{3 /\left(3+k_{E}\right)} .
$$

This is the basic result of our analysis: it gives the mass of target processed by a shock in which the fluid Mach number exceeds $\mathscr{M}_{1}$. It demontrates explicitly that, with the exception of the weakly varying factor $\sigma_{1 i}^{k_{E} /\left(3+k_{E}\right)}$ (which approaches a constant at large $\mathscr{M}_{r}$ - see eq. [2.30] below), the dynamics depends on the mass and velocity of the projectile only through the quantity $M_{P} v_{r}^{6 /\left(3+k_{E}\right)}=M_{P} v_{r}^{3 \alpha}$, as found by Dienes \& Walsh (1970) and by Rae (1970). In this form, the result applies to blast waves of arbitrary geometry (spherical, hemispherical, etc.). It is weakly dependent on the assumption that the initial collision is highly supersonic $\left(\mathscr{M}_{r} \gg 1\right)$ since, if $\mathscr{R} \neq 1$, equation (2.14) is based on that assumption; further, the argument behind equation (2.17) for $E_{i}$ is best at high $\mathscr{M}_{r}$. This result does not require $\mathscr{M}_{1}>1$, however, For the value $k_{E}=\frac{3}{8}$ which we have adopted for impact blast waves, equation (2.24) becomes

$$
\frac{M}{M_{P}}=\frac{(1+2 \mathscr{R})}{2(1+\mathscr{R})^{16 / 9}} \frac{1}{\sigma_{1 i}^{1 / 9}}\left(\frac{\mathscr{M}_{r}^{2}}{\sigma_{1} \mathscr{M}_{1}^{2}}\right)^{8 / 9} .
$$

Figure 2 portrays this result graphically.

The basic assumption underlying equation (2.24) is that the blast wave is self-similar, which will be true if $\gamma$ is constant. However, in a solid, $\gamma$ is not constant, but instead is determined by the Mach number of the shock (for $c, s$ materials, this relation is given by eq. [2.9]). We now make the quasi-steady approximation (Rae 1970, Rao 1985) and assume that the relation (2.24) remains valid for $\gamma=\gamma\left(\mathscr{M}_{1}\right)$. Rao has shown that this approximation is quite accurate.

To complete the solution, we must determine $\sigma_{1}\left(\mathscr{M}_{1}\right)$. We model the impact blast wave as part of a spherical blast wave, so that $\sigma_{1}$ is the same as that for a spherical blast wave of the same $\gamma$ and $k_{E}$. Using the "PGA" approximation developed by Ostriker \& McKee (1988), we find an approximate expression for $\sigma_{1}$, which we denote by $\sigma_{1 a}$ :

$$
\sigma_{1 a}\left(\gamma, k_{E}\right)=\frac{(\gamma+1)^{2}\left[11 \gamma-5-(\gamma+1) k_{E}\right]}{2(\gamma-1)\left[9 \gamma^{2}+20 \gamma-5+3\left(\gamma^{2}-1\right) k_{E}\right]} .
$$




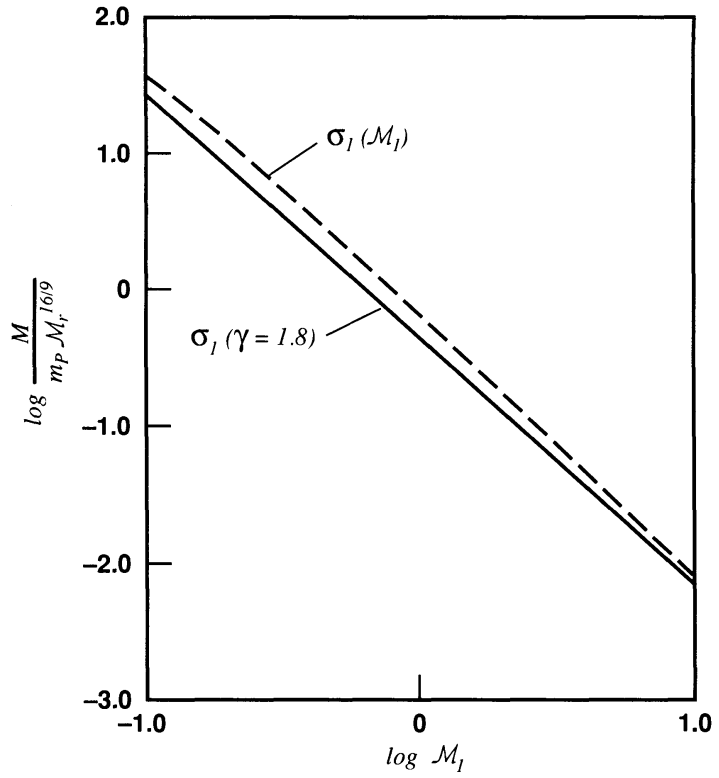

FiG. 2.- Shocked mass $M$, normalized by $M_{P} \mathscr{M}_{r}^{16 / 9}$, plotted vs. Mach number of shocked material, $\mathscr{M}_{1}$. Here, $M_{P}$ is the projectile mass and $\mathscr{M}_{r} \equiv$ $v_{r} / c_{0}$ is the Mach number defined by the relative speed $v_{r}$ of the collision partners. Like materials have been assumed $(\mathscr{R}=1)$. Solid line: $\gamma=1.8$. Dashed line: $c, s$ material with $s=1.4$, so that $\sigma_{1}$ depends on $\mathscr{M}_{1}$.

For $k_{E}=0$, this expression is exact at $\gamma=7$ and accurate to within $6 \%$ for $1 \leq \gamma \lesssim 10$; it becomes progressively less accurate for larger values of $\gamma$, however, being too low by a factor 1.8 at $\gamma=100$. For $k_{E}=\frac{3}{8}$, equation (2.26) indicates that $\sigma_{1 a}\left(\gamma, k_{E}\right)$ is within about $16 \%$ of $\sigma_{1 a}(\gamma, 0)$. It is also relatively constant, being within a factor 2 of unity throughout the range $100 \geq \gamma \geq 4 / 3$; the fact that it is relatively constant is a justification for the quasi-steady approximation. As a first approximation, one could take $\sigma_{1}$ to be constant. In order to achieve greater accuracy, we use $\sigma_{1 a}$ to infer the effect of the energy loss on $\sigma_{1}$ by writing

$$
\sigma_{1}\left(\gamma, k_{E}\right)=\left[\frac{\sigma_{1 a}\left(\gamma, k_{E}\right)}{\sigma_{1 a}(\gamma, 0)}\right] \sigma_{1}(\gamma, 0),
$$

and then obtain $\sigma_{1}(\gamma, 0)$ by numerically integrating the exact solution (Sedov 1959). We find that

$$
\sigma_{1}\left(\gamma, \frac{3}{8}\right) \simeq \frac{0.24(\gamma+0.78)^{1.3}}{\gamma-1}
$$

(Fig. 3) should be within $5 \%$ of the true value for $100 \geq \gamma \geq$ 4/3. Rao's (1985) expression is accurate for $\gamma>10$, but is too low by a factor 2 for $\gamma=4 / 3$. For the equation of state, we adopt the $c, s$ form in equation (2.8), so that

$$
\sigma_{1}\left(\mathscr{M}_{1}\right) \simeq \frac{0.30\left(s+\mathscr{M}_{1}^{-1}-0.11\right)^{1.3}}{s+\mathscr{M}_{1}^{-1}-1}
$$

from equations (2.9) and (2.27). The limiting expressions for $\sigma_{1}$ are

$$
\sigma_{1}\left(\mathscr{M}_{1}\right) \rightarrow \begin{cases}0.30(s-0.11)^{1.3} /(s-1) & \mathscr{M}_{1} \gg 1 \\ 0.30 \mathscr{M}_{1}^{-0.3} & \mathscr{M}_{1} \ll 1\end{cases}
$$

For a $c, s$ material, the shock pressure (eq. [2.7]) becomes

$$
\frac{P_{1}}{\rho_{0} c_{0}^{2}}=\mathscr{M}_{1}\left(1+s \mathscr{M}_{1}\right) \text {. }
$$

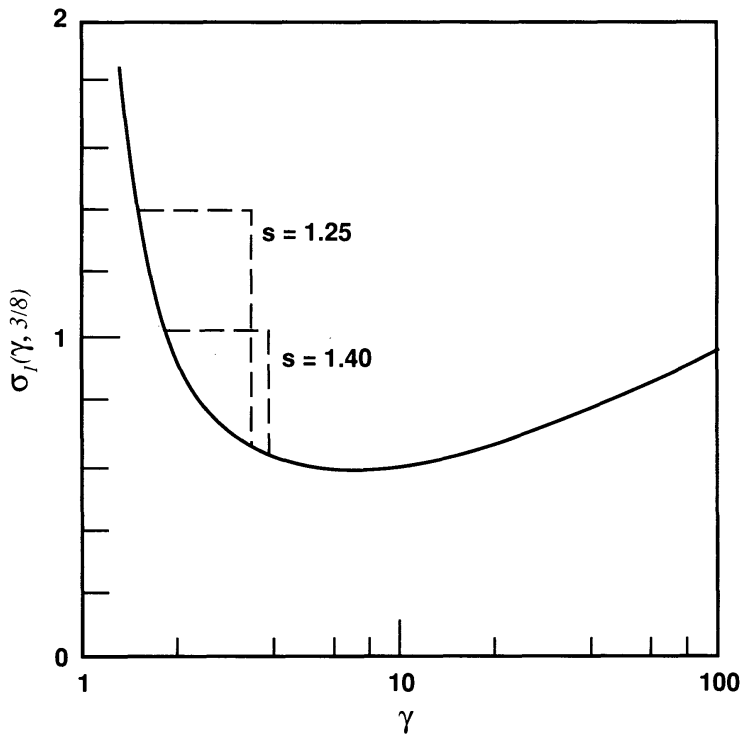

FIG. 3.-The parameter $\sigma_{1}$ (linear scale) is plotted (solid line) as a function of $\gamma$ for $k_{E}=\frac{3}{8}$. Here $\sigma_{1} \equiv E / M v_{1}^{2}$ is a constant whose value depends on the material properties of the target. For two values of the equation of state parameter $s$, the dashed horizontal lines mark the value of $\sigma_{1}$ for $\mathscr{M}_{1}=\infty$, and the vertical dashed lines mark the value of $\sigma_{1}$ for $\mathscr{M}_{1}=1$.

Since $\sigma_{1}$ is nearly constant, we can immediately determine the scaling of the pressure with radius of the blast wave. For high Mach numbers, we have $P_{1} \propto \mathscr{M}_{1}^{2} \propto M^{-9 / 8} \propto R^{-27 / 8}$, slightly steeper than $P_{1} \propto V^{-1}$. On the other hand, for low Mach numbers $P_{1}$ falls more slowly with $R: P_{1} \propto \mathscr{M}_{1} \propto M^{-9 / 16} \propto$ $R^{-27 / 16}$. Note that if the pressure is known, then the corresponding Mach number follows from equation (2.31):

$$
\mathscr{M}_{1}=\frac{2 \phi_{1}}{1+\left(1+4 s \phi_{1}\right)^{1 / 2}},
$$

where

$$
\phi_{1} \equiv \frac{P_{1}}{\rho_{0} c_{0}^{2}} .
$$

This expression, together with equations (2.25) and (2.29), determine the mass of target material shocked to a given pressure.

\subsection{Collisions at Non-normal Incidence}

In practice, grain-grain collisions will not be at normal incidence, and the theory must be extended to account for collisions at an arbitrary impact angle $\theta$ relative to the target surface. The simplest procedure would be to transform into the coordinate system in which the collision is at normal incidence $\left(v_{r} \rightarrow v_{r} \sin \theta\right)$. However, this ignores the possibility that some of the energy in the transverse motion of the target relative to the projectile will thermalize and strengthen the blast wave. We anticipate that this thermalization will be unimportant for $\mathscr{R} \gg 1$ (dense target, little penetration), but significant for $\mathscr{R} \ll 1$ (dense projectile, deep penetration).

We model a collision at an arbitrary impact angle $\theta$ by an equivalent normal collision of a particle of mass $\hat{M}_{P}$ and velocity $\hat{v}_{r}$. To allow for the possible thermalization of the relative energy, the equivalent collision has the same energy as the actual one, and we assume the transverse momentum is carried off by the exhaust, so that only the normal component of the 
momentum survives; hence,

$$
\begin{gathered}
\hat{M}_{P} \hat{v}_{r}^{2}=M_{P} v_{r}^{2}, \\
\hat{M}_{P} \hat{v}_{r}=M_{P} v_{r} \sin \theta,
\end{gathered}
$$

so that

$$
\begin{gathered}
\hat{v}_{r}=\frac{v_{r}}{\sin \theta}, \\
\hat{M}_{P}=M_{P} \sin ^{2} \theta .
\end{gathered}
$$

As discussed in $\S 2.3$, the late stage of an impact blast wave is determined by a combination of the energy and momentum and not by the velocity and mass separately, so there is no difficulty in principle in having $\hat{v}_{r} \neq v_{r}$ and $\hat{M}_{P} \neq M_{P}$.

To complete the description of the equivalent normal collision, we must specify the density of the equivalent projectile; this will determine $\widehat{\mathscr{R}}$ and hence the fraction of the collision energy which is actually transferred to the target (eq. [2.17]). Now, in a collision at non-normal incidence, the impact is spread over a larger area than in the normal incidence case. In a highly supersonic collision in which the projectile shears freely, the impact area is $\pi a_{P}^{2} / \sin \theta$, where $a_{P}$ is the radius of the projectile. We assume that the equivalent normal collision has the same impact area:

$$
\hat{a}_{P}^{2}=\frac{a_{P}^{2}}{\sin \theta} .
$$

Together with equation (2.37), this implies $\hat{\rho}=\rho \sin ^{7 / 2} \theta$ so that

$$
\hat{\mathscr{R}}=\frac{\mathscr{R}}{\sin ^{7 / 4} \theta},
$$

where we have taken $\hat{s}_{P}=s_{P}$.

The target mass shocked to a given value of $\mathscr{M}_{1}$ in a collision at an angle $\theta$ is then determined by replacing $\mathscr{M}_{r}$ by $\hat{v}_{r} / c_{0}$, etc., in equation (2.25):

$$
\frac{M(\theta)}{M_{P}}=\frac{\left(\sin ^{2} \theta+2 \mathscr{R} \sin ^{1 / 4} \theta\right)}{2\left(\sin ^{2} \theta+\mathscr{R} \sin ^{1 / 4} \theta\right)^{16 / 9}} \frac{1}{\sigma_{1 i}^{1 / 9}}\left(\frac{\mathscr{M}_{r}^{2} \sin ^{2} \theta}{\sigma_{1} \mathscr{M}_{1}^{2}}\right)^{8 / 9},
$$

from equations (2.36), (2.37), and (2.39). Since $\sigma_{1 i}$ is independent of $\mathscr{M}_{r}$ for large $\mathscr{M}_{r}$, and in any case enters to only the $\frac{1}{9}$ power, we ignore its $\theta$ dependence. The effects of non-normal incidence may be seen by comparing $M(\theta)$ with $M_{\perp}$, the value for normal incidence $(\theta=\pi / 2)$ given in eq. 2.25$)$ :

$$
\begin{aligned}
\frac{M(\theta)}{M_{\perp}}= & {\left[\left(\frac{\sin ^{2} \theta+2 \mathscr{R} \sin ^{1 / 4} \theta}{1+2 \mathscr{R}}\right)\right.} \\
& \left.\times\left(\frac{1+\mathscr{R}}{\sin ^{2} \theta+\mathscr{R} \sin ^{1 / 4} \theta}\right)^{16 / 9}\right] \sin ^{16 / 9} \theta .
\end{aligned}
$$

The factor in brackets gives the deviation from the simple coordinate transformation model in which $v_{r}$ is replaced by $v_{r}$ $\sin \theta$. First consider the case $\mathscr{R} \gg 1$ (dense target). Here, the $\mathscr{R}$ dependence cancels and $M(\theta) / M_{\perp} \simeq \sin ^{1.58} \theta$, close to the simple coordinate transformation model. Next, consider the case $\mathscr{R} \ll 1$ (dense projectile). If the deviation from normal incidence is not large $\left(\sin ^{2} \theta \gg \mathscr{R}\right)$, then $M(\theta) / M_{\perp} \simeq \sin ^{2 / 9} \theta$ : since the projectile penetrates deeply into the target, the decrease in $\boldsymbol{M}$ due to non-normal incidence is small. For glancing collisions $\left(\sin ^{2} \theta \ll \mathscr{R} \ll 1\right.$ ), the energy is dissipated close to the surface, and $M(\theta) / M_{\perp} \simeq 2 \mathscr{R}^{-7 / 9} \sin ^{1.58} \theta$ falls off fairly rapidly with decreasing $\theta$.

\subsection{Comparison to Numerical and Experimental Data}

Rae (1970) has summarized a number of numerical calculations of the variation of peak pressure with depth for highvelocity impacts. It is difficult to make a precise comparison of our results with his because ours relate the mean pressure, averaged over the surface of the shock, to the volume of shocked material. His calculations show that the shock front is somewhat elongated, with the highest velocity and pressure on the axis of symmetry. For the case which he analyzes in greatest detail, an ideal gas equation of state with $\gamma=1.5$ (corresponding to $s=1.25$ for $\mathscr{M}_{1} \gg 1$ ), the shocked volume is equivalent to that of a hemisphere with a radius equal to 0.78 times the penetration depth, and the peak pressure is about 1.5 times the mean averaged over the shock surface. The collisions are between identical materials, so that $\mathscr{R}=1$. Equation (2.28) gives $\sigma_{1}=\sigma_{1 i}=1.4$ for $\gamma=1.5$. With these values, equation (2.25) is within a factor 1.3 of Rae's " point-source similitude" prediction.

The total damage done to a solid by the impact of a projectile depends on the total energy coupled to the target as well as the decay of the pressure with distance from the impact site. In our analytical theory, the fraction, $f_{c}$, of the initial kinetic energy, which couples to the target, is given by

$$
f_{c}=\frac{(1+2 \mathscr{R})}{(1+\mathscr{R})^{2}} .
$$

(cf. eq. [2.17]) and is independent of impact velocity. This is a key assumption in our analysis. Table 2 compares this analytical result with detailed numerical calculations. In general there is a reasonable agreement, supporting our assumption. The analytical results on the dependence of the shock pressure (or shock energy) with distance from the impact point can also be compared to results from detailed numerical calculations. For a given impact velocity $v_{r}$, the shock pressure as a function of distance in the target is given by equation (2.25), using equations (2.29) and (2.32). The initial pressure is given by equation (2.16). Figure 4 compares the analytical results for $\mathrm{Fe} \rightarrow \mathrm{Fe}$ with numerical results for $\mathrm{Fe} \rightarrow \mathrm{Fe}, \mathrm{Al} \rightarrow \mathrm{Al}$, and $\mathrm{Pb} \rightarrow \mathrm{Pb}$ impacts (cf. Rae 1970). Like the numerical results, the analytical results for different materials are very similar and are for clarity not shown. A good agreement is obtained, where we note that the numerical results plot the peak pressure on the symmetry axis, which is always somewhat larger than the average shock pressure calculated by the analytical theory. The small disagreement between the analytical and numerical initial shock pressure may result from use of a slightly different equation of state. However, the difference is quite small. The decay of the shock pressure in the target is completely specified by our choice of $k_{E}$ and the numerical and analytical results for this parameter are quite similar (cf. the Appendix). It should be emphasized that there is no (further) free parameter in this particular comparison, making the agreement even more impressive. A further comparison with numerical calculations on the vapor and melt produced by impacting projectiles will be made in $\S 3$.

Last, we will here compare the analytical results for oblique impacts (cf. eq. [2.44]) with laboratory studies of cratering (Gault 1973). This comparison is somewhat hampered by the lack of knowledge on the physical parameters of these studies. Assuming for simplicity that $\mathscr{R}=1$, (i.e., like materials) we find very good agreement with the experiments further supporting our analytical approach (Fig. 5). 
TABLE 2

Comparison Between Energy Coupling in Numerical and ANalytical Studies

\begin{tabular}{|c|c|c|c|c|c|c|c|c|}
\hline \multirow[b]{3}{*}{ MATERIAL } & \multirow{2}{*}{\multicolumn{2}{|c|}{ Projectile }} & \multirow[b]{3}{*}{ MATERIAL } & & & \multicolumn{3}{|c|}{ ENERgy COUPling PARAMETER $^{a}$} \\
\hline & & & & \multicolumn{2}{|c|}{ TARGET } & \multirow{2}{*}{$\begin{array}{c}\text { Analytical } \\
f_{c}\end{array}$} & \multicolumn{2}{|c|}{ Numerical $^{\mathbf{b}}$} \\
\hline & $s_{P}$ & $\rho_{0 P}$ & & $s$ & $\rho_{0}$ & & $20 \mathrm{~km} \mathrm{~s}^{-1}$ & $72 \mathrm{~km} \mathrm{~s}^{-1}$ \\
\hline $\mathrm{Fe} \ldots \ldots \ldots \ldots$ & 1.3 & 7.9 & $\mathrm{Al}$ & 1.3 & 2.7 & 0.86 & 0.83 & 0.65 \\
\hline $\mathrm{Al} \ldots \ldots \ldots \ldots$ & 1.3 & 2.7 & $\mathrm{Fe}$ & 1.3 & 7.9 & 0.6 & 0.61 & 0.63 \\
\hline $\mathrm{p}-\mathrm{Al}^{\mathrm{c}} \ldots \ldots \ldots$ & 1.3 & 0.4 & $\mathrm{Al}$ & 1.3 & 2.7 & 0.48 & 0.56 & 0.48 \\
\hline $\mathrm{p}-\mathrm{Al}^{\mathrm{c}} \ldots \ldots \ldots$ & 1.3 & 0.4 & $\mathrm{Fe}$ & 1.3 & 7.9 & 0.33 & 0.41 & 0.38 \\
\hline
\end{tabular}

a Fraction of energy transmitted to the target (eq. [2.42]).

b Numerical data from Kreyenhagen \& Schuster 1977.

c Porous aluminum.

\section{VAPORIZATION IN GRAIN-GRAIN COLLISIONS}

The high pressures and temperatures produced upon shock compression of a material can lead to a multitude of phase transformations, including conversion from a low-pressure solid phase to a high-pressure solid phase (e.g., graphite to diamond; De Carli \& Jamieson 1961), melting, and vaporization (Zel'dovich \& Raizer 1966; McQueen et al. 1970; Al'tshuler 1965). Figure 6 shows schematically a typical phase diagram. Pressure and specific volume regimes of a lowpressure solid phase, a high-pressure solid phase, a liquid phase, and a gaseous phase as well as their mixed phases are indicated. The shock, generated by the impact of a projectile, will compress the target along the Hugoniot to a maximum pressure corresponding to the strength of the impact. Upon release by the rarefaction wave following the shock wave, the

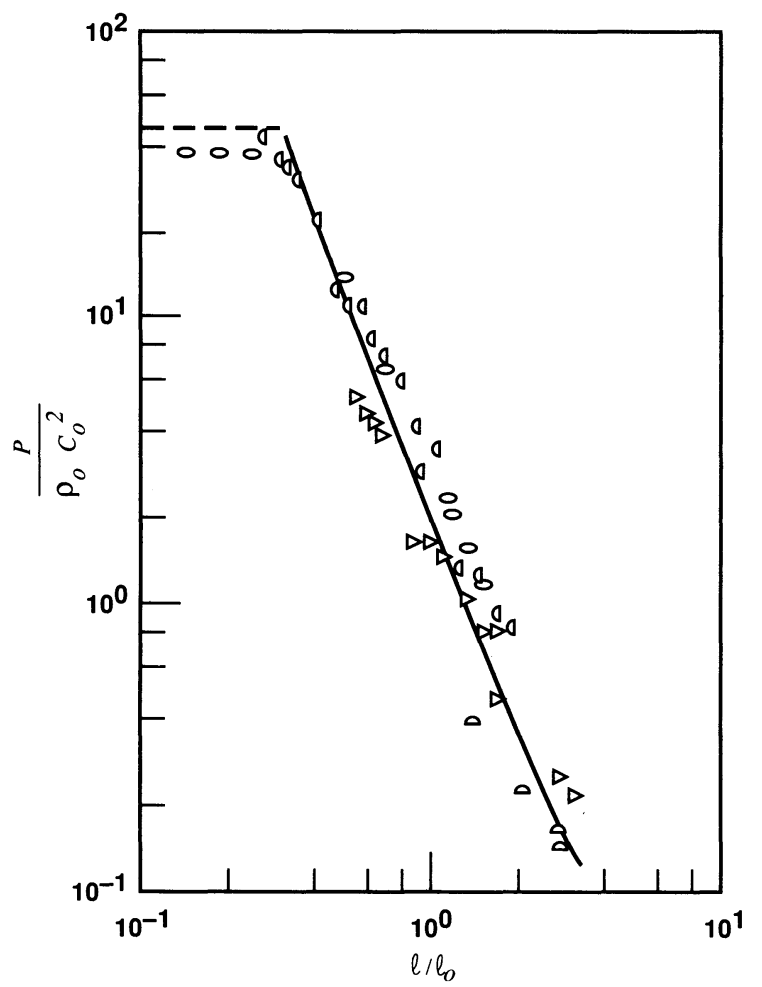

FIG. 4.-Peak pressure on the symmetry axis in units of $\rho_{0} c_{0}^{2}$ as a function of distance from the impact point, $l$ in units of the projectile size, $l_{0}$. Symbols: experimental results for $\mathrm{Fe} \rightarrow \mathrm{Fe}, \mathrm{Al} \rightarrow \mathrm{Al}$, and $\mathrm{Pb} \rightarrow \mathrm{Pb}$ impacts. Solid line: analytical results. See text for details. material will expand, and its density and temperature will continuously decrease. These release processes are virtually isentropic. Several isentropes, starting from different shock pressures, are schematically indicated in Figure 6. During this expansion the compressed material can pass through several different phase regimes, which might include the vapor or gas phase. Whether a phase transformation will actually occur depends, however, on kinetic rather than thermodynamic considerations (Zel'dovich \& Raizer 1966). This is particularly true for the microscopic size scales, and consequently very short expansion timescales $\left(\Delta t \simeq 10^{-12} \mathrm{~s}\right)$, relevant for interstellar grain collisions. For the graphite to diamond transformation this has been extensively discussed by Tielens (1990). Here we will concentrate on vaporization during the release of a compressed solid.

\subsection{Vaporization Threshold}

Although Hugoniot equations of state are generally well determined experimentally (cf. McQueen et al. 1970; Marsh 1980 ), there is a paucity of data for release isentropes. Consequently, the strength of the shock required to completely vaporize a material has to be determined largely from theoretical arguments. Above the critical point the liquid and gas phases are indistinguishable; therefore, any isentrope passing through this point or above will lead to complete vaporization of the solid. Conversely, for isentropes passing through the vapor dome only partial vaporization of the solid will take place (Zel'dovich \& Raizer 1966). The amount of vapor formed depends on the timescales involved and, if kinetic effects play

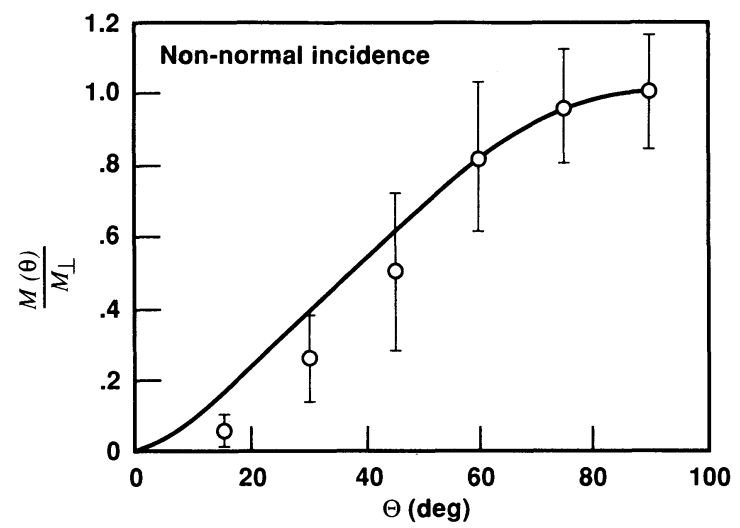

Fig. 5.- Crater mass as a function of impact angle. The crater mass is normalized to that for perpendicular impact. Symbols: experimental results; Solid line: analytical result. 


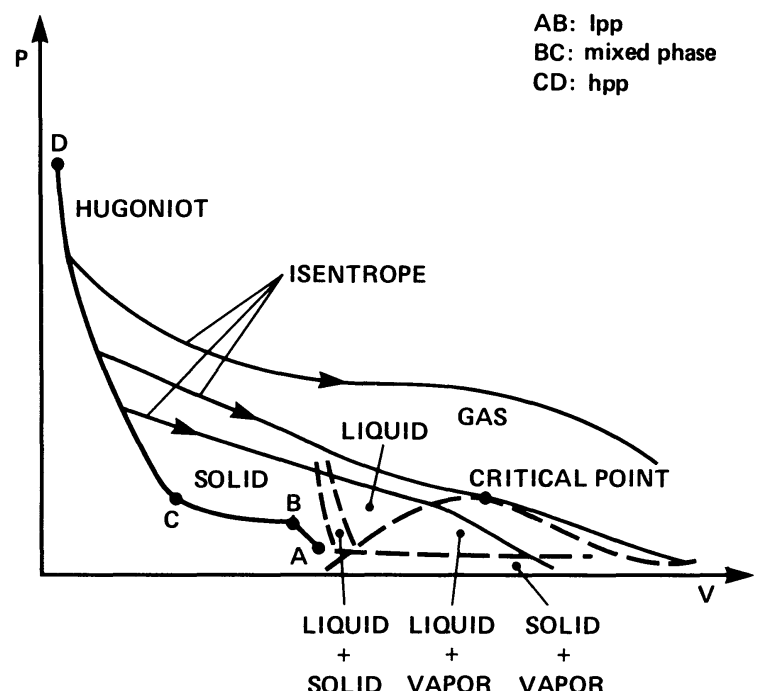

Fig. 6.-Schematic $P, V$ diagram of a realistic material. The Hugoniot of the material $(A-D)$ is the locus of states that can be reached by shocks with varying strength. After the shock has passed the material will expand along an isentrope. Several isentropes are drawn. $P, V$ regimes of various phases of the material are indicated. Besides vapor, liquid, and solid, these also include a low-pressure, high-pressure, and mixed (solid) phase (i.e., lpp, hpp, and mixed). Hence, the shock may lead to phase transformation. Complete vaporization will occur for any isentrope that passes through or above the critical point. The dashed lines enclose the vapor dome, where vapor and liquid coexist, and the region where liquid and solid coexist.

no role, is of the order of $C_{v} T_{b} / U_{0}$, where $T_{b}$ is the temperature of the released material when it crosses the boiling curve, $C_{v}$ is the heat capacity at constant volume, and $U_{0}$ is the binding energy. Let us consider as an example $\mathrm{SiO}_{2}$, which has a binding energy of about $6.5 \mathrm{eV}$ per atom. At the melting point $\left(T_{m} \simeq 2000 \mathrm{~K}\right)$, the estimated vaporized fraction in thermodynamic equilibrium is then less than $10 \%$. However, at the critical point $\left(T_{c} \simeq 13,500 \mathrm{~K}\right)$, appreciable vaporization will indeed occur. Note that upon further expansion, the vapor-solid boundary is crossed at low pressures, but kinetics will certainly prevent recondensation of the vapor on our short timescales.

Since the entropy is conserved along an isentrope, the shock pressure for complete vaporization (corresponding to the critical isentrope) can be calculated from the known entropy of the critical point (Zel'dovich \& Raizer 1966). Calculations for a variety of materials have shown that complete vaporization typically occurs when the internal energy behind the shock wave equals about 5 times the binding energy of the material (Zel'dovich \& Raizer 1966; Ahrens \& O'Keefe 1972). Partial vaporization may start at much lower shock strength, typically when the internal energy behind the shock is about equal to the binding energy of the material. However, laboratory experiments on macroscopic samples show that appreciable $(\simeq 25 \%)$ vaporization requires an internal energy behind the shock of about twice the binding energy (Hornung \& Michel 1972; Al'tshuler et al. 1977).

In order to understand this result it should be noted that the kinetic energy of the shock is partly converted into elastic and partly into thermal energy behind the shock (Zel'dovich \& Raizer 1966). The elastic component, due to compression of the solid against the repulsive interaction of the atoms, is completely transformed into kinetic energy of the material that is accelerated upon expansion. The thermal component of the internal energy behind the shock, either in the form of atomic vibrational or electronic excitation, is also partly expended in performing the work of expansion, that is, is transformed into kinetic energy. The remainder can be used for vaporization. Thus, while a shock will irreversibly increase the internal energy of the material, it is only that part of this energy that remains in the form of thermal energy after expansion to preshock densities that will lead to vaporization. As an example, consider lead compressed by a factor 1.9 to a pressure of 2.3 Mbar. Its total internal energy is about $4.7 \times 10^{10} \mathrm{ergs}^{-1}$. About $\frac{2}{3}$ of this energy is in the form of thermal (vibrational and electronic) energy. The remainder is in the form of elastic energy (i.e., compression against the repulsive interaction potential of the atoms). Upon isentropic expansion, the shocked material will pass close to the critical point and thus will be (almost) completely vaporized. After expansion to the preshock volume, the internal energy (i.e., thermal energy) of the shocked material is calculated to be about $1.9 \times 10^{10}$ ergs $\mathrm{g}^{-1}$, about twice the binding energy of lead (Zel'dovich \& Raizer 1966). Experimentally, the onset of vaporization in lead has been measured to occur for a shock pressure of $1.3 \mathrm{Mbar}$, corresponding to a total internal energy of $2.4 \times 10^{10} \mathrm{ergs} \mathrm{g}^{-1}$ (Al'tshuler et al. 1977; Asay, Trucano, \& Chhabildas 1987). As argued above, it is likely that complete vaporization will take about twice that energy, as predicted by Zel'dovich \& Raizer (1966), and thus a threshold vaporization energy of about 5 times the binding energy seems quite reasonable.

A strong shock wave generated by a high-velocity impact of a small particle on a large target will decrease in strength with depth in the target (i.e., $P \sim V^{-9 / 8}$ for $\mathscr{M}_{1} \gg 1$; see $\S 2$ ). As a result successive zones in the target will be completely vaporized, partially vaporized, molten and shattered (cf. Fig. 7). Thus, although only a limited fraction is actually vaporized,

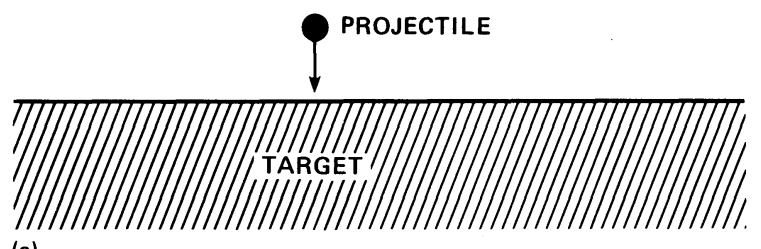

(a)

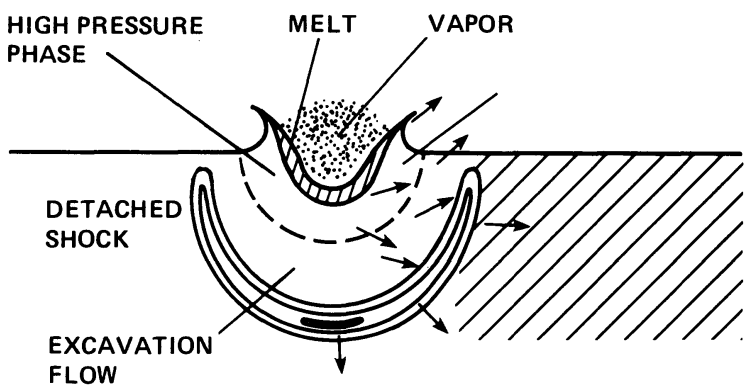

(b)

FIG. 7.-A schematic representation of the impact of a projectile on a target. The fate of the shocked target material depends strongly on the peak shock pressure. Since the strength of the shock wave driven into the target decays with distance, the impact point is surrounded by successive zones in which the target has been vaporized, molten, or converted into a high-pressure phase (e.g., diamonds). The arrows indicate the velocity vectors in the excavation flow which will carve out the final crater (see text for details). 
the volume shocked to the partial vaporization threshold is much larger than that shocked to the complete vaporization limit, and thus it may still contribute substantially to the total ! vapor produced by the impact. Rather than keeping track of the partial and complete vaporization volumes in detail, we will assume that all material shocked above a threshold pressure is completely vaporized. A threshold pressure in between the complete and partial vaporization limits will be adopted, corresponding to a total internal energy behind the shock of twice the binding energy of the material. We will compare our results to detailed numerical calculation on vaporization in impacts in $\S 3.2$.

Table 1 summarizes the material constants used to evaluate the vaporized volumes of astrophysically relevant materials. The $c_{0}$ and $s$ parameters have been determined by fitting measured Hugoniot equations of states (McQueen et al. 1970; Marsh 1980). For all materials, except graphite, typical estimates for the binding energy have been adopted. A binding energy of $4 \mathrm{eV}$ per atom has been adopted for graphite/ amorphous carbon. This is a typical value for amorphous carbon (cf. $\S 4$ ) but is considerably less than that of graphite (7.5 $\mathrm{eV}$ per atom). It is expected that soot particles formed in the outflow from C-rich giants, the main source of carbon stardust, have an hydrogenated amorphous carbon structure (Tielens 1990). This choice for the binding energy is also consistent with the threshold energy for sputtering yields measured for graphitic materials (cf. § 4). The threshold shock pressure for vaporization has then been calculated from the equation of state. The threshold collision velocity required to vaporize, calculated for like materials, is also given. The velocity behind each shock is $v_{1}=v_{1 P}=\frac{1}{2} v_{r}$ (eqs. [2.14] and [2.15]). Since the adopted threshold for vaporization is $\epsilon_{1}=2 \epsilon_{b}$ and the jump conditions give $\epsilon_{1}=\frac{1}{2} v_{1}^{2}$ (eq. [2.5]), we conclude that threshold collision velocity for like materials is $v_{t}=4 \sqrt{\epsilon_{b}}$. Note that this threshold velocity is lower (higher) for the impact of a grain with higher (lower) specific density (see $\S 2$ ).

\subsection{Comparison with Numerical Calculations}

Using a detailed numerical hydrodynamic code, O'Keefe \& Ahrens (1977) have calculated the vaporized mass produced by the impact at various velocities of silicate and iron projectiles on a semi-infinite silicate slab (i.e., gabbroic anorthosite, a wellstudied lunar highland silicate). These calculations employed an accurate equation of state, including the effects of partial and complete vaporization. The results of these calculations are compared to the semianalytical formalism (i.e., eq. [2.25] using eqs. [2.29], [2.32], and [2.33]) in Figure 8. The Hugoniot equation of state of these materials is well fitted by the $c_{0}, s$ equation (eq. [2.8]) with the parameters given in Table 2. Following O'Keefe \& Ahrens (1977), the data are plotted as a function of the parameter $S$

$$
S=\frac{\rho_{0}}{\rho_{0 P}}\left(\frac{v}{7.44 \mathrm{~km} \mathrm{~s}^{-1}}\right)^{2} .
$$

A reasonable fit to the numerical data is obtained for a threshold energy density of twice the binding energy of this silicate (i.e., $\epsilon_{v}=2 \epsilon_{b}=3.4 \times 10^{11}$ ergs $g^{-1}$ ). The corresponding threshold pressure is about $3.5 \mathrm{Mbar}$, the average of the incipient ( $\simeq 1 \mathrm{Mbar})$ and complete $(\simeq 6 \mathrm{Mbar})$ vaporization thresholds (O'Keefe \& Ahrens 1977). Both the threshold impact velocity and the absolute amount of vaporized target mass are well predicted by the analytic theory. In essence, both depend
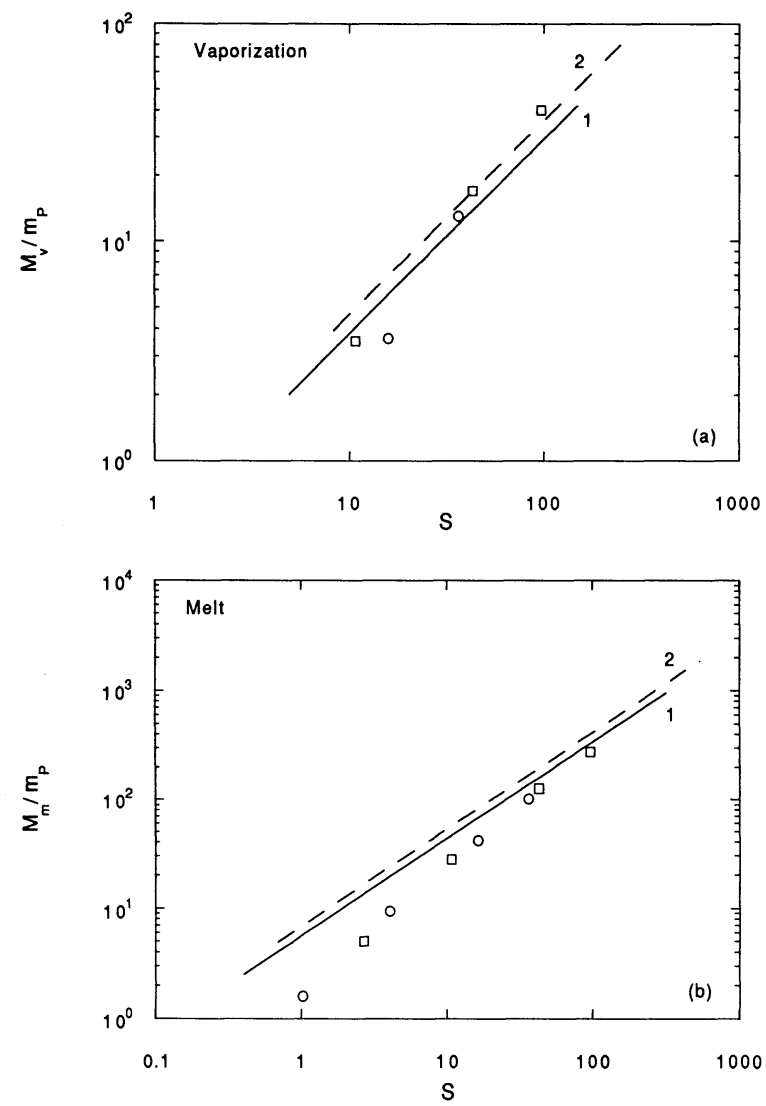

Fig. 8.-The vaporized $(a)$ and molten $(b)$ target mass in units of the projectile mass as a function of the shock strength parameter, S. Solid line: analytical results for anothosite on anorthosite. Dashed line: analytical results for iron on anorthosite. Data points: numerical calculations (O'Keefe \& Ahrens 1977). Circles: anorthosite on anorthosite. Squares: iron on anorthosite. See text for details.

directly on the assumed value of the threshold pressure. We can also compare the predictions for the analytic theory with numerical calculations for the melt mass generated by an impact (O'Keefe \& Ahrens 1977). In this case, the threshold pressures for incipient and complete melting, 430 and $520 \mathrm{kbar}$ respectively, are very similar and this free parameter is eliminated. Assuming a threshold pressure of $520 \mathrm{kbar}$, a good fit to the data is obtained at high impact pressures, but near threshold the analytical theory overestimates the molten mass considerably (Fig. 8). Intuitively, it is expected that at threshold a target mass equal to the projectile mass is shocked to the threshold pressure, and the numerical results seem to bear this out. The reason for this is that the pressure is relatively uniform during the first stage of the collision. However, the analytic theory was derived for the late stage, in which the blast wave is decelerating and the energy is concentrated near the surface. From equation (2.22), using equations (2.14) and (2.17) we find

$$
\frac{M_{i}}{M_{P}}=\frac{1+2 \mathscr{R}}{2 \sigma_{1 i}} .
$$

The value of $\sigma_{1 i}$ can be determined from equations (2.29) and (2.32). For silicates, we find $\sigma_{1 i}=0.58$ for $P_{1}=520 \mathrm{kbar}$. As a result, equation (3.2) predicts $M_{1} \simeq 2.6 M_{P}$ at threshold, which is too large by a factor of 2.6. However, for grain-grain collisions in interstellar shocks, we are mainly interested in 
high-velocity impacts of small projectiles on large targets (i.e., $P \gg P_{t}$ ), and this mismatch near threshold is of little concern as long as the threshold pressure has been chosen appropriately. Finally, we emphasize, once more, that kinetic considerations may to some extent inhibit vaporization on the short timescales involved in interstellar grain collisions. Hence, although we reproduce these macroscopic calculations well, our general choice for the threshold vaporization energy of twice the binding energy is perhaps on the low side.

\subsection{Application to Grain-Grain Collisions in Interstellar Shocks}

In order to estimate the vaporized volume due to grain-grain collisions in interstellar shocks three steps have to be taken. First, for a given size ratio of projectile and target, the vaporized (diamondized, molten, or shattered) volume is calculated as a function of collision velocity, taking the finite size of the grains into account. Second, this volume is averaged over impact angle since glancing collisions couple less of the projectile energy and momentum to the shock wave in the target (cf. $\S 2.4)$. Third, this average volume has to be integrated over the actual collision velocity distribution in a shock. In particular, since grains are spiraling around the interstellar magnetic field, at any one point in the shock, there is a range of probable collision velocities between two grains. The first two steps will be taken here, and an approximate, but simple, analytical formula for the average vaporized volume will be derived as a function of collision velocity. This can then be used as input for a numerical code which calculates the structure of an interstellar shock wave.

Consider the collision of two grains with radii $\left(a_{P}\right.$ and $\left.a_{T}\right)$ at a relative velocity $v$ and impact angle $\theta$. At threshold $\left(v=v_{t}\right)$ only the target material in the first stage (cf. $\S 2$ ) is vaporized, since the pressure in the late stage blast wave is too low for vaporization. This threshold velocity is given by

$$
v_{t}=(1+\mathscr{R})\left(2 \epsilon_{v}\right)^{1 / 2},
$$

where $\epsilon_{v}$ is the specific vaporization energy (Table 2). In this limit, the fraction vaporized in terms of projectile mass, $f_{v 1}$, is approximately given by

$$
f_{v 1} \equiv \frac{M_{v 1}}{M_{P}}=\frac{1}{2}(1+2 \mathscr{R}) \frac{h_{\text {over }}}{\sigma_{1 i}},
$$

which follows directly from equation (3.2). The factor $h_{\text {over }}$ approximately corrects the collision energy for the average overlapping volume and is given by

$$
h_{\mathrm{over}}=\left(1+\frac{a_{p}}{a_{T}}\right)^{-1} .
$$

At high impact velocities the vaporized volume (for a semiinfinite slab) can be found by averaging equation (2.41) over the impact angle. To a good approximation this average is given by $1.55 \mathscr{R}^{0.18}$ for $0.5 \leq \mathscr{R} \leq 2$ and the vaporized target mass relative to the projectile mass, $f_{v 2}$, is then given by

$$
f_{v 2} \equiv \frac{M_{v 2}}{M_{P}}=0.78 \frac{1+2 \mathscr{R}}{(1+\mathscr{R})^{16 / 9}} \frac{1}{\sigma_{1 i}^{1 / 9}}\left(\frac{\mathscr{M}_{r}^{2}}{\sigma_{1} \mathscr{M}_{1}^{2}}\right)^{8 / 9} \mathscr{R}^{0.18}
$$

valid when $v \gg v_{t}$. The two limits, $f_{v 1}$ and $f_{v 2}$, can be combined into one equation for the vaporized target mass, $M_{v}$, in terms of the total target mass, $M_{T}$,

$$
F_{v} \equiv \frac{M_{v}}{M_{T}}=\frac{f}{1+f}
$$

where $f$ is given by

$$
f=\left[f_{v 1}+f_{v 2}\left(1-\frac{v_{t}}{v}\right)^{1 / 2}\right] \frac{M_{P}}{M_{T}},
$$

for $v \geq v_{t}$ the threshold velocity. The factor $(1+f)$ has been inserted into equation (3.7) to ensure that $F_{v} \leq 1$. When considering the vaporized mass of the projectile (i.e., the smaller of the two colliding bodies) the ratio of projectile to target mass in the equation for $f$ should be set equal to unity. This analytical approximation for the relative vaporized volumes is compared with actual numerical integration of the relevant formulae for the case of graphite grain-grain collisions in Figure 9. Comparisons like these show that this relatively simple analytical approximation for the vaporized mass fraction as a function of impact velocity reproduces the numerical results reasonably well (within a factor 1.5 ) for a variety of material parameters. They hold for collisions of like and unlike grain materials as well as for target and projectile. Finally, they can also be used to calculate the fraction of a grain molten or shattered by an impact by inserting the appropriate threshold pressure and velocity for these processes.

Most previous studies of vaporization upon grain-grain collision (Oort \& van de Hulst 1946; Barlow 1978b) assumed that, in a head-on collision, vaporization will occur when the kinetic energy of relative motion is equal to the binding energy of the grains, that is,

$$
v_{t}=\left(M_{T}+M_{P}\right)\left(\frac{2 \epsilon_{b}}{M_{T} M_{P}}\right)^{1 / 2} .
$$

For equal-sized grains this reduces to $v_{t}=\sqrt{8 \epsilon_{b}}$, a factor $\sqrt{2}$ less than our assumed threshold velocity, reflecting the difference in the assumed threshold internal energy. Of course, this formula does not provide a good estimate of the threshold velocity for unequal-sized grains. Seab \& Shull (1983) in their analysis of the effects of a size distribution on grain-grain collisions assumed a threshold of about $v_{t}=\sqrt{6 \epsilon_{b}}$ for equal-sized grains. For the head-on collision of two grains with widely disparate masses, the smaller one was assumed to vaporize at a threshold velocity of $v_{t} \simeq \sqrt{3 \epsilon_{b}}$, which underestimates the threshold velocity considerably. The larger one was assumed

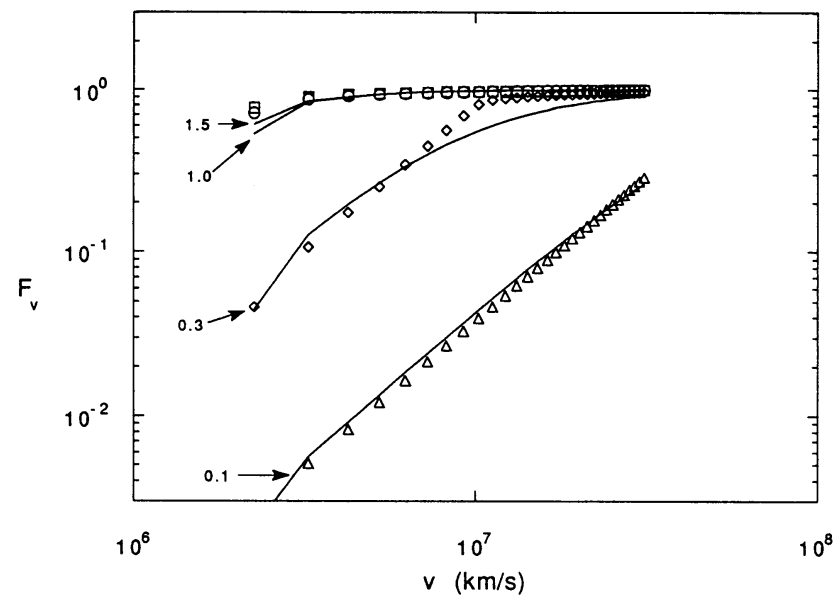

FIG. 9.-The vaporized target mass in terms of the total target mass as a function of impact velocity for various target to projectile size ratios. Symbols: numerical evaluation of the averaging over impact angle taking the finite size of the grain into account (see $\S 3.3$ ). Solid lines: analytical approximation (eq. [3.7]) 
to vaporize completely at a threshold velocity larger by the square root of the mass ratio. For typical interstellar grain size ratios $(\simeq 10)$ and binding energies $(\simeq 5 \mathrm{eV}$ per atom), this threshold velocity is $\simeq 350 \mathrm{~km} \mathrm{~s}^{-1}$, and such high-velocity collisions are very rare in the ISM. In our analysis, total vaporization in a single collision will occur at a similar high threshold velocity for widely disparate masses. However, the impact of a small grain at velocities in excess of the threshold velocity $\left(\simeq 20 \mathrm{~km} \mathrm{~s}^{-1}\right.$ ) will lead to partial vaporization, albeit only a small mass fraction (Fig. 9).

Consider now a MRN grain size distribution [i.e., $n(a) d a \sim a^{-3.5} d a$; Mathis, Rumpl, \& Nordsieck 1977). Small grains are rapidly stopped in an interstellar shock, and, thus, the collision velocity with a large target is approximately the same (i.e., the target velocity) for all projectiles. For an infinite slab, the vaporized volume scales with the projectile volume, and hence the largest projectiles will dominate the vaporization. For a finite target with size $a_{T}$, vaporization will be dominated by those projectiles which are just big enough to completely destroy the target. For a $100 \mathrm{~km} \mathrm{~s}^{-1}$ collision velocity, this corresponds to projectiles with radius $\simeq 0.3 a_{T}$ (see Fig. 9). For this example, half the vaporized volume is then contributed by projectiles with sizes in the range $\simeq 0.075-0.3 a_{T}$, or $200-750 \AA$ for the largest interstellar grain targets. For higher collision velocities, this shifts to smaller sizes.

Finally, it is of some interest to compare the efficiency of shattering with that of vaporization by grain-grain collisions. For macroscopic brittle bodies, shattering is dominated by the initiation, growth, and coalescence of cracks. As a result, low velocity collisions $\left(\simeq 10 \mathrm{~m} \mathrm{~s}^{-1}\right)$ can lead to shattering. However, for submicron-sized bodies, cracks play little role, and the strength of a material approaches the ultimate yield strength of the material, typically $\simeq 100 \mathrm{kbar}$ (Jones et al. 1994). This corresponds to an energy of $2 \times 10^{8} \mathrm{ergs}^{-1}$ or a threshold velocity of $0.4 \mathrm{~km} \mathrm{~s}^{-1}$. Indeed, extensive experimental studies of the micrometeorite hazard on spacecraft show that crater formation commences at about this threshold velocity (cf. Jones et al. 1994 and references therein). Hence, for an infinite target, the volume shattered is about $10^{3}$ times larger than the volume vaporized. For a relative velocity of 100 $\mathrm{km} \mathrm{s}^{-1}$, a finite target is completely shattered for a projectile to target size ratio larger than 0.025 , or $60 \AA$ for a $2500 \AA$ target. Thus essentially, any grain-grain collision at this velocity will lead to the complete disruption of the target. ${ }^{4}$

\section{SPUTTERING OF INTERSTELLAR GRAINS}

Behind a strong shock gaseous ions impacting a grain at high velocities will sputter off atoms from the surface layer (cf. Seab 1987). For astrophysical purposes, the gas can be considered to consist of $\mathrm{H}, \mathrm{He}$, and the $\mathrm{CNO}$ group ions. For low-velocity, radiative shocks $\left(v_{s} \leq 200 \mathrm{~km} \mathrm{~s}^{-1}\right)$, betatronaccelerated grains are moving at high velocity with respect to the gas. This leads to so called "nonthermal" sputtering. Because of its larger mass (i.e., energy), nonthermal sputtering by $\mathrm{He}^{+}$dominates over that by $\mathrm{H}^{+}$. For high-velocity, nonradiative shocks $\left(v_{s} \geq 200 \mathrm{~km} \mathrm{~s}^{-1}\right)$, grains are not betatron accelerated but rather are dragged to rest with respect to the gas before the postshock gas cools and compresses. In that case, sputtering is dominated by the thermal motion of ions in

\footnotetext{
${ }^{4}$ The projectile is of course completely vaporized in such an impact.
}

the gas (i.e., thermal sputtering) and sputtering by $\mathrm{H}^{+}$is the most important because of its higher abundance. Sputtering by the CNO group is only important near or below the threshold for sputtering by $\mathrm{He}$ and $\mathrm{H}$. Previous studies of sputtering of grains in interstellar shocks have been presented by Draine \& Salpeter (1979b), Barlow (1978a), Shull (1978), and Seab \& Shull (1983). This discussion improves on these studies by including the results from recent experimental and theoretical studies. Thorough reviews of the present understanding of sputtering of solids by ions have been presented in the books edited by Behrisch $(1981,1983)$. Of particular interest are the chapters by Andersen \& Bay (1981) and Betz \& Wehner (1983) who present extensive compilations of experimental data pertaining to sputtering of single-element and multicomponent materials, respectively. Our theoretical understanding of sputtering has been summarized by Sigmund (1981). Semiempirical sputtering theories have been presented by Bohdansky, Roth, \& Bay (1980), Bohdansky (1980), Matsunami et al. (1980), Yamamura, Matsunami, \& Itoh (1983), and Bohdansky (1984). We will follow the last study to arrive at a "universal " sputtering relation and apply it to astrophysically relevant grain materials.

\subsection{Sputtering Yields}

High-energy sputtering can be treated by a linear cascade process, which views the event as a series of binary collisions between atoms in the solid (Sigmund 1981). As a result of these collisions, some recoiling surface atoms receive sufficient energy and momentum to overcome the surface binding forces. The total sputtering yield, $Y$, for normal incident projectiles impacting with energy, $E$, is then given by

$$
Y(E)=4.2 \times 10^{14} \frac{\alpha S_{n}(E)}{U_{0}} \text { atoms per ion },
$$

where $\alpha$ is an energy independent function of the mass ratio between target and projectile, $U_{0}$ is the surface binding energy (in units of $\mathrm{eV}$ ), and $S_{n}(E)$ is the so-called nuclear stopping cross section (in units of ergs $\mathrm{cm}^{2}$ ). Essentially, the sputtering yield depends on the ratio of the deposited energy per unit length, which is proportional to $N \alpha S_{n}(E)$ with $N$ the volume density (atoms $\mathrm{cm}^{-3}$ ), to the surface binding energy of the target (Sigmund 1981). The nuclear stopping cross section can be expressed in the "universal" relation (Sigmund 1981),

$$
S_{n}(E)=4.2 \pi a Z_{1} Z_{2} e^{2} \frac{M_{1}}{M_{1}+M_{2}} s_{n}\left(\epsilon_{12}\right),
$$

where $M_{i}$ and $Z_{i}$, are the projectile $(i=1)$ and target $(i=2)$ atomic masses and numbers. The screening length for the interaction potential between the nuclei, $a$, is given by

$$
a=0.885 a_{0}\left(Z_{1}^{2 / 3}+Z_{2}^{2 / 3}\right)^{-1 / 2},
$$

where $a_{0}$ is the Bohr radius (0.529 $\AA$ ) and the universal function, $s_{n}\left(\epsilon_{12}\right)$, depends on the detailed form adopted for the screened Coulomb interaction. Detailed numerical and analytical studies (Matsunami et al. 1980) show that this function can be approximated by

$$
S_{n}\left(\epsilon_{12}\right)=\frac{3.441 \sqrt{\epsilon_{12}} \ln \left(\epsilon_{12}+2.718\right)}{1+6.35 \sqrt{\epsilon_{12}}+\epsilon_{12}\left(-1.708+6.882 \sqrt{\epsilon_{12}}\right)},
$$


where $\epsilon_{12}$ is given by

$$
\epsilon_{12}=\frac{M_{2}}{M_{1}+M_{2}} \frac{a}{Z_{1} Z_{2} e^{2}} E .
$$

The dimensionless function $\alpha$ depends only on the ratio $M_{2} / M_{1}$ (and the angle of incidence). In the range $0.5<$ $M_{2} / M_{1}<10, \alpha$ can be approximated by

$$
\alpha=0.3\left(M_{2} / M_{1}\right)^{2 / 3},
$$

while for $M_{2} / M_{1}<0.5, \alpha$ is approximately constant $(\simeq 0.2)$. This increase of $\alpha$ with increasing mass ratio results from the increased importance of backscattered projectile ions to the ejection of surface target atoms (Sigmund 1981). For large mass ratios $\left(M_{2} / M_{1}>5\right)$, this expression gives unreasonably large values for $\alpha$ (Andersen \& Bay 1981). This is partly due to the neglect of electronic stopping, which is relatively more important for light ions, and partly to errors in the deposited energy distribution function. For simplicity, the latter is generally evaluated assuming that the surface does not influence the development of the collision cascade (i.e., assuming an infinite target). In the case of light projectiles, this leads to an unrealistically large number of multiple scattering events in the target and consequently overestimates the deposited energy in the surface layer. To first order, this can be corrected for by multiplying by the ratio of the mean projected range to the mean penetrated path length, $R_{p} / R$, which can be approximated by (Bohdansky 1984)

$$
\frac{R_{p}}{R}=\left(K \frac{M_{2}}{M_{1}}+1\right)^{-1}
$$

For elastic collisions, $K$ is only weakly dependent on $\epsilon$ and about equal to 0.4 . When electronic stopping is included, the dependence of $K$ on $\epsilon$ becomes more pronounced (Sigmund 1981). We will assume $K$ is independent of $\epsilon$ and treat it as a free parameter. Near threshold, sputtering is dominated by recoiling projectile ions which transfer sufficient energy and momentum to surface target atoms on their way out to induce target erosion. The threshold energy, $E_{\mathrm{th}}$, for sputtering is then given by (Bohdansky et al. 1980; Andersen \& Bay 1981)

$$
\begin{gathered}
E_{\mathrm{th}}=\frac{U_{0}}{g(1-g)} \text { for } \frac{M_{1}}{M_{2}} \leq 0.3, \\
E_{\mathrm{th}}=8 U_{0}\left(M_{1} / M_{2}\right)^{1 / 3} \text { for } M_{1} / M_{2}>0.3,
\end{gathered}
$$

with the maximum fractional energy transfer possible in a head-on elastic collision, $g$, given by

$$
g=\frac{4 M_{1} M_{2}}{\left(M_{1}+M_{2}\right)^{2}} .
$$

The equation for $E_{\mathrm{th}}$ at small mass ratios follows directly from energy and momentum conservation. At high mass ratios, a fit to an extensive set of experimental data has been employed (Bohdansky et al. 1980; Andersen \& Bay 1981). At low projectile energies, a large collision cascade does not develop and a considerable number of the recoiling target atoms receive insufficient energy to overcome the surface binding energy. This effect can be corrected for by replacing the deposited energy by an "effectively deposited energy". This results in substituting $S_{n, 1}$ for $S_{n}$ in equation (4.1), where the former is given by (Bohdansky et al. 1980; Yamamura et al. 1983)

$$
S_{n, 1}=S_{n}\left[1-\left(\frac{E_{\mathrm{th}}}{E}\right)^{2 / 3}\right] \text {. }
$$

The assumption of an isotropic momentum distribution, used in deriving these sputtering yield equations, also breaks down at low projectile energies. This leads to a similar correction factor (Bohdansky 1984)

$$
S_{n, 2}=S_{n, 1}\left(1-\frac{E_{\mathrm{th}}}{E}\right)^{2} .
$$

Near threshold $\left(E_{\mathrm{th}}<E \ll 20 E_{\mathrm{th}}\right)$, the energy dependence of the sputtering yield is mainly determined by these two factors. This theoretical relation is very similar to a widely used empirical relation and gives a surprisingly good fit to a wide range of experimental data (Bohdansky et al. 1980; Andersen \& Bay 1981; Bohdansky 1984). The final formula for the sputtering yield at normal incidence is then given by

$$
\begin{aligned}
Y(E)=\frac{3.56}{U_{0}(e V)} \frac{M_{1}}{M_{1}+M_{2}} \frac{Z_{1} Z_{2}}{\sqrt{Z_{1}^{2 / 3}+Z_{2}^{2 / 3}}} \alpha \frac{R_{p}}{R} s_{n}(\epsilon) \\
\times\left[1-\left(\frac{E_{\mathrm{th}}}{E}\right)^{2 / 3}\right]\left(1-\frac{E_{\mathrm{th}}}{E}\right)^{2} \text { atoms per ion }
\end{aligned}
$$

valid for $E>E_{\mathrm{th}}$.

Finally, more energy is deposited in the surface layer for glancing impacts, resulting in an increased sputtering yield. For very large angle of incidence $\left(\theta>80^{\circ}\right)$, the probability of direct reflection of the incident ion from the surface becomes very large and the deposited energy and thus the sputtering yield will decrease again. Theoretical and experimental studies suggest an approximate $1 / \cos \theta$ dependence for the sputtering yield between $\left(0 \leq \theta \leq 80^{\circ}\right.$ : Sigmund 1981; Andersen \& Bay 1981). This increases the angle averaged sputtering yield by a factor of 2 from that for normal incidence (Draine \& Salpeter 1979a). For the surface binding energy, $U_{0}$, we will adopt the sublimation energy of the material. The only free parameter in the expression for the sputtering yield is then the constant $K$ which enters into the expression for $R_{p} / R$, which we will determine from a comparison with laboratory studies of relevant materials. This is discussed in the remainder of this section. The adopted values are summarized in Table 3.

\subsection{Sputtering Parameters for Selected Materials}

\subsubsection{Graphite}

Because of its importance in nuclear technology, the interaction of energetic particles with graphite has been studied in great detail. Recent reviews of the literature have been given by Roth (1983) and Auciello, Haasz, \& Stangeby (1985). Sputtering of graphite is influenced by two factors: Chemical sputtering and radiation damage. First, at high enough target temperatures $(\simeq 800 \mathrm{~K})$, chemical sputtering is important. The interaction of energetic (as well as sub-eV) atomic hydrogen with an aromatic carbon network gives rise to hydrocarbon formation at active surface areas (i.e., edges of the basal plane). These hydrocarbons rapidly desorb. Besides the formation of hydrocarbons, chemical sputtering is characterized by a pronounced temperature dependence and is of little importance below room temperature (Behrisch et al. 1976; Yamada et al. 1980; Roth et al. 1976; Roth, Bohdansky, \& Wilson 1982; 
TABLE 3

SPUTTERING OF INTERSTELLAR GRAINS

\begin{tabular}{|c|c|c|c|c|c|c|c|c|}
\hline \multirow[b]{2}{*}{ MATERIAL } & \multicolumn{2}{|c|}{ SURFACE LAYER $^{a}$} & \multicolumn{4}{|c|}{ YIELD PARAMETERS $^{b}$} & \multirow[b]{2}{*}{ GAS } & \multirow[b]{2}{*}{ REFERENCES } \\
\hline & $\begin{array}{c}d \\
(\AA)\end{array}$ & Composition & $\begin{array}{c}U_{0} \\
(\mathrm{eV})\end{array}$ & $\begin{array}{c}M_{2} \\
(\mathrm{amu})\end{array}$ & $Z_{2}$ & $K$ & & \\
\hline 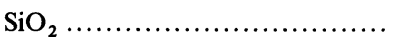 & 10 & $\mathrm{Si}$ & 6.4 & 20 & 10 & 0.1 & c & $1,2,3,4$ \\
\hline Silicate $\ldots \ldots \ldots \ldots \ldots \ldots \ldots \ldots \ldots$ & 10 & $\mathrm{Fe}$ & 5.7 & 23 & 11 & 0.1 & $\ldots$ & 5 \\
\hline Graphite/amorphous carbon ..... & 10 & $\ldots$ & 4 & 12 & 6 & 0.65 & d & $6,7,8,9,10$ \\
\hline 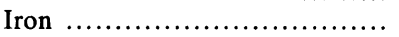 & $\ldots$ & $\ldots$ & 4.1 & 56 & 26 & 0.35 & $\ldots$ & $6,14,15$ \\
\hline
\end{tabular}

${ }^{\text {a }}$ In all cases the surface layer is amorphitized by ion bombardment (see text). The value of $d$ lists the depth of penetration for $\mathrm{H}$ atoms impacting at $100 \mathrm{~km} \mathrm{~s}^{-1}$. Helium atoms at this velocity penetrate about a factor of 2 deeper. After the bombardment the surface layer is enriched in the element listed under composition.

b Parameters used to evaluate the sputtering yields (cf. $\S 4$ ). Only $K$ is a "truly" free parameter.

c Gaseous products: $\mathrm{Si}, \mathrm{O}, \mathrm{SiO}, \mathrm{SiO}_{2}, \mathrm{Si}_{2} \mathrm{O}, \mathrm{Si}_{2}$.

d Gaseous products: $\mathrm{CH}_{4}, \mathrm{C}_{2} \mathrm{H}_{2}, \mathrm{C}_{2} \mathrm{H}_{4}$

e Gaseous products: $\mathrm{H}_{2} \mathrm{O}, \mathrm{H}, \mathrm{O}, \mathrm{H}_{3} \mathrm{O}^{+}$.

REFERENCES.- (1) Roth el al. 1979; (2) Bach 1970; (3) Bach et al. 1974; (4) Edwin 1973; (5) Our estimate; (6) Behrisch et al. 1976; (7) Roth et al. 1976; (8) Bohdansky et al. 1978; (9) Yamada et al. 1980; (10) Hechtl et al. 1981; (11) Brown et al. 1980; (12) Bar-nun et al. 1985; (13) Rocard et al. 1986;(14) von Seefeld et al. 1976;(15) Borders et al. 1978.

Davis, Haasz, \& Stangeby 1987). In the initial phase of the exposure the chemical sputtering yield shows a maximum which decreases subsequently to a steady state value. Probably, precursor states to the hydrocarbon formation process give rise to $\mathrm{CH}_{n}$ surface species, which when occurring at adjacent surface sites block further reaction with $\mathrm{H} \mathrm{(a}$ "deactivated surface"; Gould 1975).

Second, at high enough fluences $\left(>10^{15}\right.$ ions $\left.\mathrm{cm}^{-2}\right)$, ion bombardment of graphite produces pronounced radiation damage, such as bond angle disorder, vacancies and interstitial carbon atoms. This leads to a reduction in the crystallite size of the aromatic network and eventually to a complete amorphization of the lattice. The structural rearrangement of the carbon lattice due to ion implantation influences the surface binding energy and thus the sputtering yield. Indeed, at a fluence of about $10^{18}$ ions $\mathrm{cm}^{-2}$, Raman spectroscopy, transmission electron microscopy, resistivity measurements, and $\mathrm{X}$-ray photoelectron spectroscopy of ion-bombarded graphite reveal the characteristics of amorphous carbon over the full implanted range (Wright, Varma, \& Gruen 1976; Elman et al. 1984; Ashida et al. 1984, 1986; Venkatesan et al. 1984). As discussed below, the fluences in interstellar shocks can easily exceed values of $10^{18}$ ions $\mathrm{cm}^{-2}$, transforming graphite surfaces into amorphous carbon.

Finally, the situation is even more complicated by synergism between these two effects. Simultaneous exposure to energetic Ar ions and sub-eV $\mathrm{H}$ atoms leads to a drastic enhancement of the sputtering yield (Vietzke, Flaskamp, \& Phillipps 1984). Apparently, energetic ion bombardment facilitates the precursor states to $\mathrm{CH}_{3}$ formation by reaction of loosely bound $\mathrm{C}$ atoms at vacancies or interstitials with $\mathrm{H}$ present on the surface or implanted during previous processing. Alternatively, the bombardment with energetic ions may remove the siteblocking complexes, which would also result in an enhanced sputtering yield.

Given this complexity, it is not surprising that published results on the sputtering of carbon materials span several orders of magnitude reflecting differences in sample conditioning, vacuum environment, sample structure, and measurement techniques (Roth 1983; Auciello et al. 1985). For example, mea- surements reported by one group on two carbon samples supplied by two different companies using the same experimental apparatus nevertheless differed by a factor 10 in the sputtering yield (Behrisch et al. 1976). For these reasons care should be taken in evaluating the sputtering yield of graphitic materials in interstellar space from laboratory studies. Fortunately, in the interstellar medium, dust temperatures are generally between 10 and $100 \mathrm{~K}$, even in shocked regions, and chemical sputtering is of no direct concern (Draine 1979). Likewise, simultaneous high-energy ion and sub-eV $\mathrm{H}$ bombardment is likely to be unimportant.

One important aspect of shock processing of interstellar carbon grains is that the surfaces of these grains are almost certain to resemble hydrogenated amorphous carbon due to the bombardment by ions in shocks. This is true irrespective of the original structure of the carbon dust (graphite, diamond). Ignoring betatron acceleration, a grain will encounter its own weight in colliding gas ions behind the shock. Hence, the total fluence, $F$, is approximately given by

$$
F \simeq \frac{a \rho_{0}}{m_{\mathrm{H}}} \simeq 10^{19} \frac{a}{1000 \AA} \text { ions } \mathrm{cm}^{-2}
$$

This is likely to be an underestimate of the actual dose. The projected range of $100 \mathrm{~km} \mathrm{~s}^{-1} \mathrm{H}$ and $\mathrm{He}$ ions $(\simeq 50$ and 200 $\mathrm{eV}$ ) is approximately 10 and $20 \AA$, corresponding to the outermost five basal planes (Sigmund 1981; Burenkov 1986). The hydrogen concentration in these outermost layers, which dominate the sputtering of grains, can easily saturate at these fluences. Thus, these layers will be highly disordered and completely hydrogenated $(\mathrm{H} / \mathrm{C} \simeq 0.4)$ by this ion bombardment, and we expect their sputtering yields to be similar to the physical sputtering yields measured for deactivated carbon surfaces in the laboratory studies discussed above. As an aside we note that ion bombardment and $\mathrm{H}$ implantation into diamonds will also lead to a hydrogenated amorphous carbon surface layer and thus the sputtering yield of diamond grains is expected to show a very similar behavior to that measured for graphite. Adopting the MRN interstellar dust model with a total carbon-grain surface area of $\simeq 10^{-21} \mathrm{~cm}^{-2}$ per $\mathrm{H}$ atom and a 
hydrogenated amorphous carbon surface layer of thickness 10 $\AA$, about $3 \%$ of the elemental $\mathrm{C}$ is locked up in these shockproduced hydrogenated amorphous carbon (HAC) mantles. This is quite comparable to the observed fraction of elemental C locked up in hydrocarbon mantles in the diffuse interstellar medium as deduced from the $3.4 \mu \mathrm{m}$, aliphatic $\mathrm{C}-\mathrm{H}$ stretching, absorption feature (Pendleton et al. 1994). While laboratory-produced HAC does not provide a perfect fit to the shape of this absorption feature, the presence of a small amount of electronegative impurities (such as $\mathrm{OH}$ ) may improve the fit considerably (Pendleton et al. 1994), and such impurities may result directly from the implantation process. Hence, it is conceivable that the $3.4 \mu \mathrm{m}$ absorption feature signals the presence of shock processed aromatic carbon surfaces in the interstellar medium 5 .

The formation of an amorphous carbon surface layer will reduce the surface binding energy. This is partly a result from the change in bonding character (i.e., planar vs. tetrahedral) and partly from the presence of dangling bonds and aliphatic carbon chains (e.g., saturated with $\mathrm{H}$ ), which are only "loosely" incorporated into the solid network. Typically, a carbon binding energy of only about $4 \mathrm{eV}$ is expected (Benson 1976) rather than the $7.5 \mathrm{eV}$ per $C$ atom in graphite ${ }^{6}$. Adopting this surface binding energy, a good fit to the measured sputtering yields of $\mathrm{H}, \mathrm{D}$, and $\mathrm{He}$ ions on the basal plane of pyrolytic graphite at or below room temperature (Roth et al. 1976; Bohdansky, Bay, \& Ottenberg 1978; Yamada et al. 1980) can be obtained for $K=0.65$ (cf. Fig. 10). Similar yields have been measured for hydrogenated amorphous carbon films, a-C:H (Davis \& Haasz 1987), and glassy carbon (Yamada et al. 1980). The surface binding energy of amorphous carbon substrates derived this way are in good agreement with those $(\simeq 4 \mathrm{eV})$ derived from erosion measurements due to ion $(\mathrm{H}, \mathrm{D}, \mathrm{He}, \mathrm{Ar})$ bombardment at high target temperatures $(>2000 \mathrm{~K})$. At such high temperatures, evaporation and annealing of active states leads to an enhanced sputtering yield (Phillips, Flaskamp, \& Vietzke 1982; Roth et al. 1982) and an independent measurement of the surface binding energy can be obtained this way. In contrast, using the graphite binding energy of $7.5 \mathrm{eV}$ per atom, the experimental graphite data is rather poorly fitted by the semiempirical formulae described in $\$ 4.1$ (Andersen \& Bay 1981; Strazzula, Calcagno, \& Foti 1985). Much higher yields as well as a higher energy threshold have been reported for evaporated carbon on platinum using Auger spectroscopy (Smith, Meyer, \& Layton 1976, 1977). However, these results are probably strongly influenced by backscattering from the heavy substrate atoms, leading to overestimates of the sputtering yield particularly at higher energies (Andersen \& Bay 1981). Likewise, early sputtering measurements (Rosenberg \& Wehner 1962) of $\mathrm{He}^{+}$on graphite using plasma discharge techniques are marred by experimental difficulties (Andersen \& Bay 1981), and we have disregarded those results as well. Finally, mass spectrometer studies of the sputtering products resulting from $\mathrm{H}$ bombardment show that, although initially

\footnotetext{
${ }^{5}$ Carbon stardust, like soot produced by hydrocarbon flames, is expected to have a $H / C$ ratio of $\simeq 0.1$ (Tielens 1990 ). However, such low $H$ fractions will not lead to $\mathrm{HAC}$ formation and, since the $\mathrm{C}-\mathrm{H}$ bonds are aromatic (i.e., benzene-like) rather than aliphatic (ie., $\mathrm{CH}_{2}$ and $\mathrm{CH}_{3}$ groups), these grains will not show a $3.4 \mu \mathrm{m}$ feature.

${ }^{6}$ This reduction of the binding energy of carbon solids is a consequence of the variety of chemical valence structure and bonding possible with carbon. Although other materials may amorphitize under ion bombardment, no large reduction of their binding energy is expected.
}

$\mathrm{CH}_{3}$ is the dominant product, on its diffusion outward this radical is saturated with chemisorbed atomic $\mathrm{H}$ to form $\mathrm{CH}_{4}$ (cf. Bohdansky \& Roth 1985; Vietzke et al. 1984). Some heavier hydrocarbons are released as well. In particular, at lower energies $(E \simeq 100 \mathrm{eV})$ comparable amounts of $\mathrm{C}_{2} \mathrm{H}_{2}$ and $\mathrm{C}_{2} \mathrm{H}_{4}$ have been detected (Phillipps \& Vietzke 1987; Yamada 1987).

\subsubsection{Silicates}

Experimental studies of the sputtering yield of silicates have been limited to $\mathrm{SiO}_{2}$. The available data on sputtering by $\mathrm{He}$ and $\mathrm{Ar}$ ions is summarized in Figure 10. Again, older data using plasma discharges have been ignored. As was the case for carbon discussed in $\S 4.2$, ion bombardment of oxides, such as glasses, increases the disorder by broadening the $\mathrm{Si}-\mathrm{O}$ bond angle distribution function. At a high enough dose, an amorphitized surface layer will result (Kelly et al. 1972; Kelly 1984). This is, however, not expected to lead to a drastic reduction of the surface binding energy. For the surface binding energy we will follow Kelly \& Lam (1973) and adopt the "average" heat of atomization, $\Delta H_{a}$ : that is,

$$
\mathrm{SiO}_{2}(s)=\mathrm{Si}(g)+2 \mathrm{O}(g)
$$

and

$$
\Delta H=3 \Delta H_{a} \text {. }
$$

Adopting a mean mass and atomic number of $20 \mathrm{amu}$ and 10 , respectively, and the heat of atomization of $\mathrm{SiO}_{2}(6.4 \mathrm{eV}$ per atom), these data are fitted reasonably well by the general sputtering formulae for $K$ equal to 0.1 (cf. Table 3). Perhaps the slightly more rapid fall-off with decreasing ion energy near threshold indicates a somewhat higher surface binding energy.

The discussion in $\S 4.1$ is, strictly speaking, applicable only to single-element materials. For multicomponent materials, such as silicates, nonstochiometric removal of surface atoms is generally of importance. Due to differences in mass and surface binding energy, different components in a solid will have different sputtering yields. This, as well as recoil implantation and thermal and radiation-induced segregation, will produce a surface layer with an altered composition (Betz \& Wehner 1983). Due to the complex interplay of these effects, the outcome cannot be predicted a priori. For many oxides, such as $\mathrm{SiO}_{2}$, the high vapor pressure component (i.e., O) will be preferentially lost upon sputtering. The depth of this stochiometrically altered layer is comparable to the ion range of the incident ion. At low temperatures (i.e., thermal diffusion is unimportant), a steady state can be reached where the sputtered material has bulk composition. Typically, this requires removal of a surface layer comparable in size to the ion range. In a typical interstellar shock $\left(v \simeq 100 \mathrm{~km} \mathrm{~s}^{-1}\right) \mathrm{H}$ and $\mathrm{He}$ atoms are implanted in silicates to a depth of about 10 and 20 $\AA$, respectively, corresponding to a volume fraction of about $0.05(1000 \AA / a)$. Comparing this to the volume fraction sputtered in this shock (McKee et al. 1987), we conclude that such a dynamical equilibrium will be reached for a grain size larger than about $300 \AA$ in a single $100 \mathrm{~km} \mathrm{~s}^{-1}$ shock. The sputtering yields for a Si-rich surface layer are (of course) very similar to those measured for $\mathrm{SiO}_{2}$ (Betz \& Wehner 1983). Finally, both secondary ion and neutral mass spectrometry studies have shown that sputtering of $\mathrm{SiO}_{2}$ leads to the ejection of small molecules (e.g., $\mathrm{SiO}, \mathrm{Si}_{2}, \mathrm{SiO}_{2}$, and $\mathrm{Si}_{2} \mathrm{O}$ ) as well as atoms. For $\mathrm{Ar}^{+}$ions bombardment at $330 \mathrm{eV}$, comparable amounts of atomic and molecular species have been detected (Oeschner 1985). 

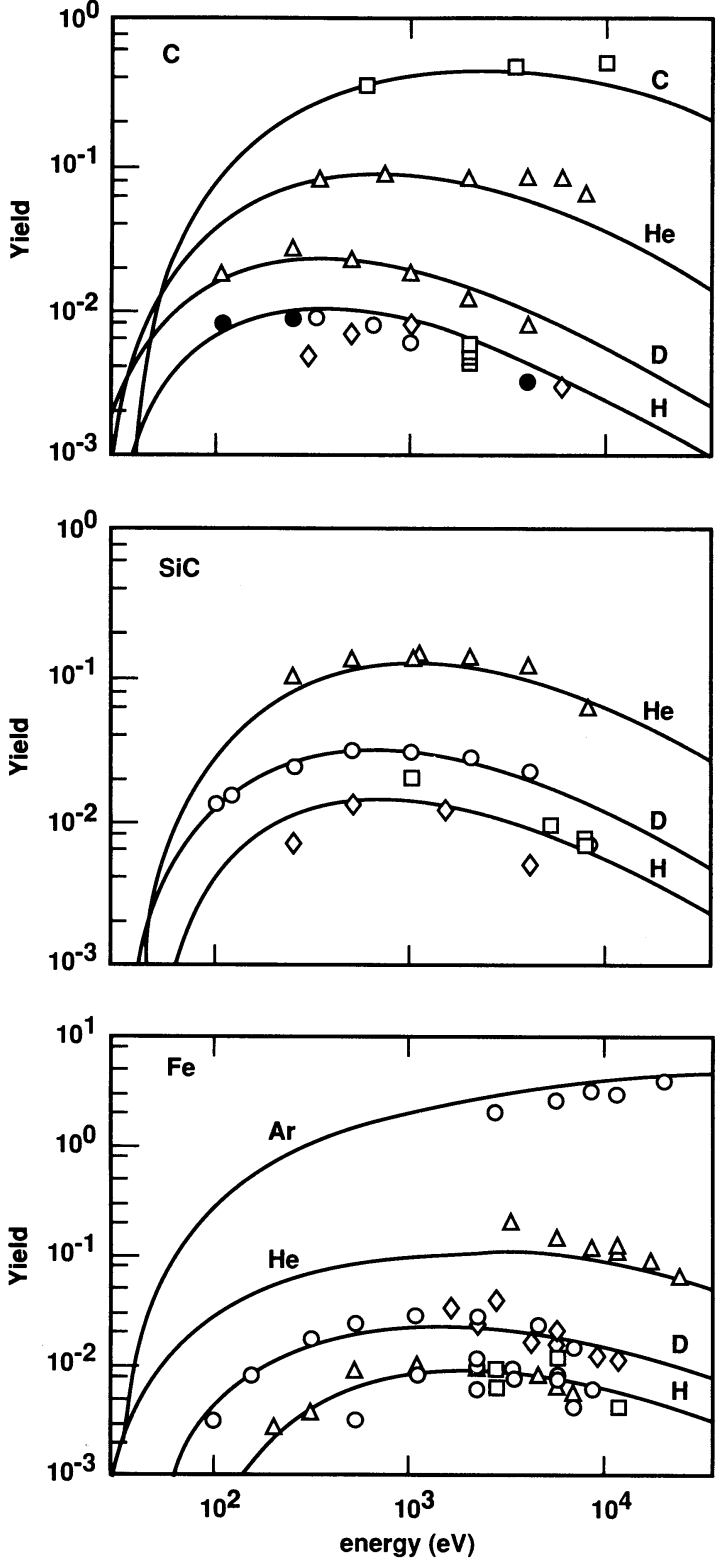

Interstellar silicates are generally thought to contain appreciable amounts of $\mathrm{Fe}$ and $\mathrm{Mg}$ with an approximate chemical formula $(\mathrm{Fe}, \mathrm{Mg}) \mathrm{SiO}_{4}$. To the best of our knowledge, no experimental data on the sputtering yield of such silicates have been reported in the literature. In our studies of sputtering of interstellar silicates (Jones et al. 1993a), we will adopt an average binding energy of $5.7 \mathrm{eV}$ per atom, a value of 0.1 for the constant $K$ (as for $\mathrm{SiO}_{2}$ ), and an average atomic mass and atomic number of $23 \mathrm{amu}$ and 11, respectively. Interstellar silicates are generally thought to be highly amorphous as a result of their formation under supercooled conditions (Tielens \& Allamandola 1987; Tielens 1990). Ion bombardment in interstellar shocks will only increase this disorder further. Furthermore, the surface layer may be enriched in $\mathrm{Fe}$ due to nonstochiometric sputtering (Yin, Ghose, \& Adler 1972). In fact, the presence of finely divided metallic iron in the lunar soil has been attributed to extensive solar wind bombardment (Pillinger, Gardiner, \& Jull 1976). This is of some importance
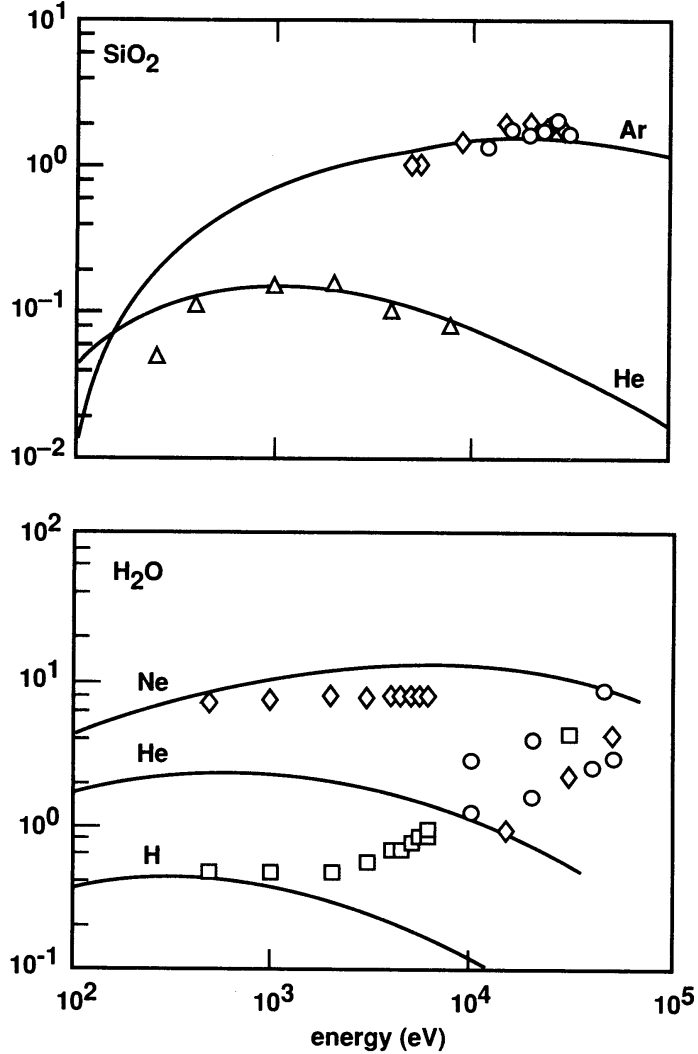

FIG. 10.-Comparison of the theoretical sputtering yields with experimental data. References to the experimental data are given in Table 3. Top left: $\mathbf{H}^{+}$ (triangles and crosses), $\mathrm{D}^{+}$( filled circles), $\mathrm{He}^{+}$(squares), and $\mathrm{C}^{+}$(open circles) impacts on graphite. Similar sputtering yields are obtained in experimental studies of amorphous carbon. Note that the data indicated by crosses measure only $\mathrm{CH}_{4}$ (and $\mathrm{CH}_{3}$ ) production. The yield of heavier carbon molecules is however appreciable at lower energies (Phillipps \& Vietzke 1987; Yamada 1987), and they should be assigned less weight in determining the threshold energy. Top right: $\mathrm{He}^{+}$(squares) and $\mathrm{Ar}^{+}$(triangles) impacts on $\mathrm{SiO}_{2}$. Middle left : $\mathrm{H}^{+}$(triangles), $\mathrm{D}^{+}$(circles), and $\mathrm{He}^{+}$(squares) impacts on silicon carbide Bottom right: $\mathrm{H}^{+}$(triangles and crosses), $\mathrm{He}^{+}$(squares), and $\mathrm{Ne}^{+}$(circles) impacts on $\mathrm{H}_{2} \mathrm{O}$. The rise in sputtering yields for energies above about $10 \mathrm{keV}$ are due to electronic energy losses (see text). Bottom left: $\mathrm{H}^{+}$(triangles, squares, circles), $\mathrm{D}^{+}$(diamonds, circles), $\mathrm{He}^{+}$(triangles), and $\mathrm{Ar}^{+}$(circles) impacts on iron.

since the superparamagnetic properties of this single-domain surface layer may lead to efficient magnetic alignment of interstellar silicate grains. Yet, the wavelength dependence of linear and circular polarization will be dominated by the silicate core, a dielectric material, rather than the thin (10-20 $\AA)$ metallic iron grain mantle, a highly absorbing material. This may explain one of the most puzzling aspects of interstellar polarization studies, the presence of a very well aligned, yet transparent, interstellar dust component. The presence of an iron surface layer may also be of interest from a chemical point of view. Fischer-Tropsch reactions on such surfaces may convert appreciable amounts of $\mathrm{CO}$ into larger hydrocarbon molecules at elevated temperatures $(>300 \mathrm{~K})$.

\subsubsection{Silicon Carbide}

As for graphite, extensive studies have been made of the sputtering of silicon carbide due to its possible importance as an inner liner in tokamak devices. The experimental data on $\mathbf{H}$, 
$\mathrm{D}$, and He sputtering are summarized in Figure 10. The adopted value for the "average" heat of atomization of $\mathrm{SiC}$ is $6.3 \mathrm{eV}$ per atom. A very good fit of the "universal" sputtering relation to this data is obtained, assuming $K$ is equal to 0.3 . Like $\mathrm{SiO}_{2}$, the lighter component (i.e., C) is preferentially sputtered from the surface layers (Betz \& Wehner 1983). Again, ion bombardment will lead to an amorphous surface layer. While $\mathrm{SiC}$ grains are not abundant in the interstellar medium, the extraction of presolar $\mathrm{SiC}$ grains from carbonaceous meteorites makes studies of their survival in the interstellar medium very opportune (Anders et al. 1989).

$$
\text { 4.2.4. Iron }
$$

The experimental data on $\mathrm{H}, \mathrm{D}, \mathrm{He}$, and Ar sputtering of iron (stainless steel) is summarized in Figure 10. The adopted value for the "average" heat of atomization of $\mathrm{Fe}$ is $4.1 \mathrm{eV}$ per atom. A very good fit of the "universal" sputtering relation to this data is obtained, assuming $K$ is equal to 0.35 . Some blister formation occurs in the sputtering of iron when the implanted gas concentration exceeds a critical value of about 0.3 gas atom per target atom. However, at the relevant impact energies ( $<10 \mathrm{keV}$ ), this blistering is not accompanied by exfoliation (flaking of the surface layers), and sputtering dominates the mass loss (Behrisch et al. 1976).

\subsubsection{Mantle Material}

Two kinds of grain mantles are thought to exist in interstellar space: (1) icy grain mantles and (2) organic refractory grain mantles (Greenberg 1982; Tielens \& Allamandola 1987). Icy grain mantles contain predominantly volatile molecules such as $\mathrm{H}_{2} \mathrm{O}, \mathrm{CO}$, and $\mathrm{CH}_{3} \mathrm{OH}$, and we will approximate their sputtering by that of its dominant component (i.e., $\mathrm{H}_{2} \mathrm{O}$ ). Only a limited amount of data on sputtering of water ice at low ion energies are available (Fig. 10). A reasonable fit can be obtained for $K$ is equal to 0.1 . Note that at high energies $(E>10 \mathrm{keV})$ sputtering is dominated by electronic ionization and excitation of target molecules. This is not included in the theoretical description in $\S 4$. However, such high-energy sputtering is of little importance in sputtering of interstellar grains, and we will ignore it. Because of its low formation temperature, interstellar icy grain mantles are expected to be highly amorphous (cf. Hagen, Tielens, \& Greenberg 1983; Tielens \& Allamandola 1987), and ion bombardment in interstellar shocks has probably little further effect in this respect. Ion bombardment of mixed molecular ices containing appreciable amounts of carbonaceous molecules, such as $\mathrm{CO}$ and $\mathrm{CH}_{3} \mathrm{OH}$, will lead to the formation of a non-volatile carbonaceous surface layer (Strazulla et al. 1985; Lanzerotti, Brown, \& Johnson 1985), which may protect the underlying ice against sputtering. However, it is expected that UV photons, which have a much larger penetration depth, will be more important in converting ices into a refractory, carbonaceous residue in the interstellar medium. Prolonged UV processing of interstellar grain mantles may eventually lead to the formation of an amorphous carbon grain mantle (Tielens 1989; Jenniskens et al. 1993). We will approximate the sputtering of such an organic refractory grain mantle by that of amorphous carbon. Finally, the dominant gaseous products of sputtering of mixed, volatile ices are the individual molecular components. Some ejection of atomic and molecular products of ion bombardment (i.e., $\mathrm{H}, \mathrm{O}, \mathrm{H}_{2}$, $\mathrm{O}_{2}$, and $\mathrm{H}_{3} \mathrm{O}^{+}$; see Johnson et al. 1985) have also been measured, but their yield depends sensitively on diffusion processes, and low-temperature $(\simeq 10 \mathrm{~K})$ studies have not yet been performed.

\subsection{Summary of Nonthermal Sputtering of Interstellar Grains}

The non-thermal sputtering rate, $d N_{\mathrm{sp}} / d t$, of a grain moving at velocity $v_{g}$ through the gas is given by

$$
\frac{d N_{\mathrm{sp}}}{d t}=2 \pi a^{2} v_{g} \sum n_{i} Y_{i}\left(E=0.5 m_{i} v_{g}^{2}\right)
$$

where the factor 2 takes nonnormal incidence into account, and $n_{i}$ is the density of ions $i$ with mass $m_{i}$. The rate of decrease of grain size is then given by

$$
\frac{d a}{d t}=\frac{m_{\mathrm{sp}}}{2 \rho_{0}} v_{g} n_{\mathrm{H}} \sum A_{i} Y_{i}\left(E=0.5 m_{i} v_{g}^{2}\right),
$$

with $m_{\mathrm{sp}}$ and $\rho_{0}$ the average mass of the sputtered atoms and the specific density of the grain material, and $A_{i}$ as the abundance of ion $i$.

Figure 11 compares the sputtering yield for $\mathrm{He}^{+}$impacting astrophysically relevant materials. As discussed before, organic refractory grain mantles and diamond grains will probably have sputtering yields very similar to those of amorphous carbon. Not very surprising, as a result of the lower binding energy of $\mathrm{H}_{2} \mathrm{O}$ compared to refractory materials (i.e., 0.5 vs. 5 $\mathrm{eV})$, the threshold energy is an order of magnitude lower and the yield is an order of magnitude larger than for other materials (cf. eq. [4.13]). Icy grain mantles will thus easily be sputtered even in low-velocity shocks (Draine \& Salpeter 1976b). Except near threshold, all refractory materials have very similar sputtering yields (to within a factor 2), reflecting their similarity in binding energy. In particular, this holds also for silicates and (amorphous) carbon grains (cf. Struzulla, Baratta, \& Magazzu 1985). In contrast, some previous studies used the binding energy of graphite to estimate the sputtering yield of interstellar graphite grains (Seab \& Shull 1983) and consequently underestimated the sputtering yield considerably, in particular at the lower energies. Of all the materials considered, $\mathrm{SiC}$ has the highest sputtering threshold, as a result of its larger binding energy.

Comparing our sputtering yields to previous studies, we find that our thresholds for silicates and graphite are very similar to those of Draine \& Salpeter (1979b). However, our sputtering yields rise somewhat more steeply with increasing velocity (or energy), amounting to a factor 3-4 higher sputtering yield for velocities between 30 and $\simeq 100 \mathrm{~km} \mathrm{~s}^{-1}$. For high velocities $\left(v>100 \mathrm{~km} \mathrm{~s}^{-1}\right)$, Draine \& Salpeter $(1979 \mathrm{~b})$ adopt similar

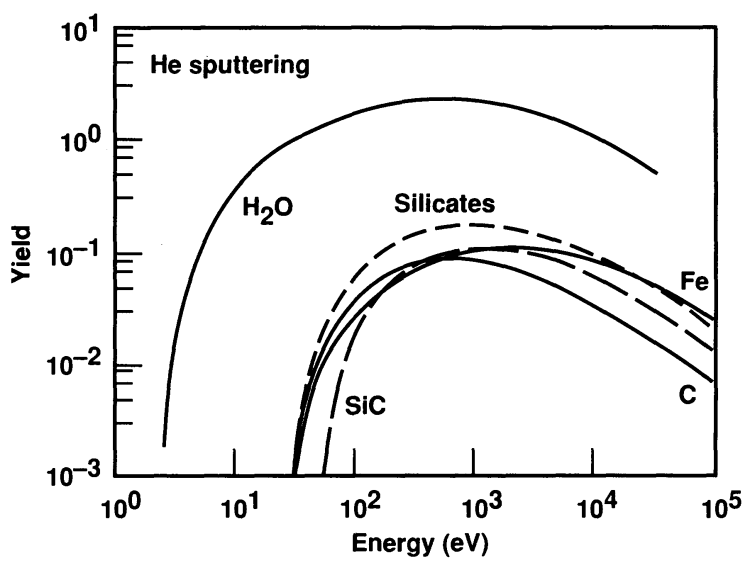

FIG. 11.-Comparison of the theoretical sputtering yields of $\mathrm{He}^{+}$ions incident on astrophysically relevant materials. 
sputtering yields for graphite and silicate grains. These differ from our values by a factor 2 . Seab \& Shull adopt much higher sputtering thresholds, and their sputtering yields are a factor of 10 lower for low velocities $\left(v<100 \mathrm{~km} \mathrm{~s}^{-1}\right)$. At high velocities, their graphite sputtering yields agree with ours to within $20 \%$, but their silicate yields are a factor 2 higher. Our theoretical fits to the experimental data are to be preferred. The experimental data base for carbon (and $\mathrm{SiC}$ ) is quite substantial, and our derived sputtering yields are very reliable (better than $30 \%$; see Fig. 10) over the whole energy range. In contrast, the yields for interstellar silicates have been derived from the scarce data for $\mathrm{SiO}_{2}$, and their uncertainty is larger, $50 \%$. Moreover, the binding energy of interstellar silicates is not well known, and hence the uncertainty near threshold is much larger. The adopted value is, however, reasonable.

\subsection{Summary of Thermal Sputtering of Interstellar Grains}

At high temperatures, sputtering by (thermally) impacting ions can become of importance. We have therefore evaluated the thermal sputtering rate using the newly derived sputtering yields. The sputtering rate is now given by

$$
\frac{d N_{\mathrm{sp}}}{d t}=2 \pi a^{2} \sum n_{i}\left\langle Y_{i} v\right\rangle
$$

where $\left\langle Y_{i} v\right\rangle$ is the sputtering yield of ion $i$ averaged over the Maxwellian distribution and $n_{i}$ is the density of ion $i$. The factor 2 takes again the average of the yield over angles of incidence into account. The rate of decrease in size, $d a / d t$, is then given by

$$
\frac{1}{n_{\mathrm{H}}} \frac{d a}{d t}=\frac{m_{\mathrm{sp}}}{2 \rho_{0}} \sum A_{i}\left\langle Y_{i} v\right\rangle,
$$

where $m_{\mathrm{sp}}$ and $\rho_{0}$ are the average mass of the sputtered atoms and the specific density of the grain material, and $A_{i}$ is the abundance of ion $i$. In our numerical evaluation of equation (4.20), we have taken impacts by $\mathrm{H}, \mathrm{He}, \mathrm{C}, \mathrm{N}$, and $\mathrm{O}$ into account.

Figure 12 shows the calculated thermal sputtering rates (eq. [4.20]) as a function of temperature in units of $\mathrm{cm}^{3} \AA \mathrm{yr}^{-1}$. The sputtering rates for graphite and silicates are very similar and rise steeply with increasing temperature. Typically, thermal sputtering will be important for temperatures greater than $\simeq 10^{6} \mathrm{~K}$, corresponding to shock velocities $>200 \mathrm{~km} \mathrm{~s}^{-1}$ (Seab 1987). In our evaluation the grain charge has not been taken into account, which can be of some importance for $T$ less than $10^{5} \mathrm{~K}$. For such low temperatures, the grain charge is a complicated function of such parameters as the incident and shock-generated UV field and the electron density (McKee et al. 1987). Inclusion of these effects would introduce some (small) substructure in the thermal sputtering curves at low

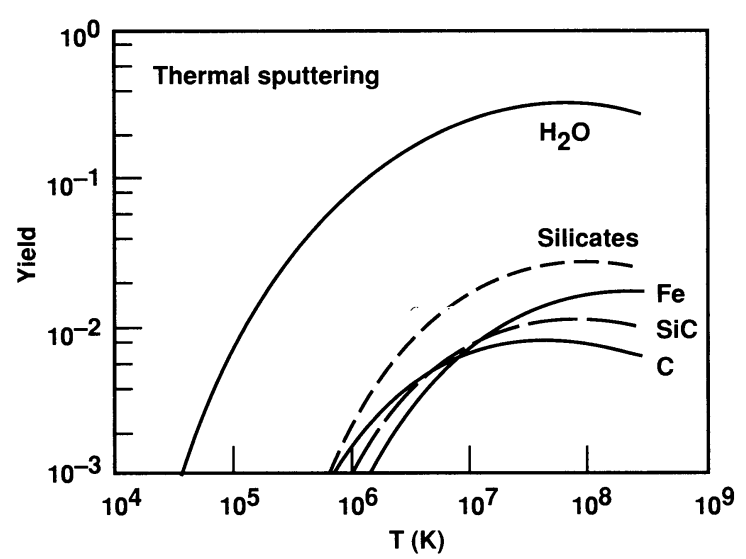

FIG. 12.-Thermal sputtering rate, $\left(1 / n_{\mathrm{H}}\right) /(d a / d t)$ in units $\mathrm{of}^{3} \AA \mathrm{yr}^{-1}$ for various astrophysically relevant materials. Parameters for these fifth-order polynomial fits are given in Table 4.

temperatures but would not affect the overall characteristics (cf. Draine \& Salpeter 1979b). These thermal sputtering curves have been fitted by fifth-order polynomials

$$
y=\sum_{i=0}^{5} a_{i} x^{i},
$$

where $x$ and $y$ are the $\log$ (base 10) of $T$ in units of $10^{6} \mathrm{~K}$ and $\left(1 / n_{\mathrm{H}}\right) d a / d t$, respectively. The coefficients $a_{i}$ are listed in Table 4 , and those polynomials are valid in the range $10^{4}$ $\left(3 \times 10^{8}\right) \mathrm{K}$

\section{SUMMARY}

We have examined the physical processes that destroy dust grains in interstellar shocks. Grain-grain collisions in shocks drive strong shock waves in the grains. Upon unloading, these shock waves can lead to vaporization of the material. We have derived an analytical model for the propagation of strong waves in solids in order to determine the fraction of the mass shocked to pressures exceeding the vaporization limit. The results for this analytical model agree very well with detailed numerical calculations, supporting our analysis. We have derived simplified analytical formulae (eqs. [3.3]-[3.8]) which give the fraction of a grain vaporized in a collision as a function of impact velocity, projectile mass, and material parameters. Based upon theoretical and experimental evidence, the threshold pressure required to vaporize target material is determined. This threshold pressure corresponds to an internal energy behind the shock of about twice the binding energy. For refractory materials, (partial) vaporization of a grain will occur for collision velocities in excess of $20 \mathrm{~km} \mathrm{~s}^{-1}$. Likely, vaporization by grain-grain collisions in interstellar shocks is domi-

\begin{tabular}{|c|c|c|c|c|c|c|}
\hline Material & $a_{0}$ & $a_{1}$ & $a_{2}$ & $a_{3}$ & $a_{4}$ & $a_{5}$ \\
\hline Silicate $\ldots \ldots \ldots \ldots \ldots \ldots \ldots \ldots$ & -2.7446 & 1.5439 & -0.37046 & 0.21641 & -0.34755 & 0.10114 \\
\hline Graphite/amorphous carbon ...... & -2.8605 & 1.0572 & -0.27545 & 0.23735 & -0.31820 & 0.087376 \\
\hline 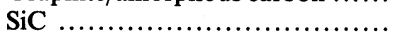 & -3.0862 & 1.3984 & -0.4119 & 0.3438 & -0.3778 & 0.0990 \\
\hline Ice $\ldots \ldots \ldots \ldots \ldots \ldots \ldots \ldots$ & -1.1023 & 0.7074 & -0.2041 & 0.04298 & -0.05124 & 0.0140 \\
\hline Iron & -3.2944 & 1.4978 & -0.2614 & 0.2447 & -0.3338 & 0.0898 \\
\hline
\end{tabular}

TABLE 4

THERMAL SPUTtering FITs ${ }^{a}$

a Coefficients in the fifth-order polynomial fit to the thermal sputtering rates. See text for details. 
nated by the high-velocity impact of medium-sized grains on large grains (projectile-to-target size ratio $\simeq 0.3$ ). In general, we predict that vaporization is much less important than previously considered (Seab \& Shull 1983). The theory for the propagation of shock waves in solids can also be used to determine the fraction of a grain that is transformed into a higher density phase or shattered by a collision. The former has been used by Tielens et al. (1987) to determine the fraction of carbonaceous grains transformed into diamonds by grain-grain collisions in shocks. Because the limiting shattering pressure ( $\simeq 100 \mathrm{kbar}$; Tielens 1989$)$ is much less than for vaporization ( $\simeq 5$ Mbar), low-velocity collisions $\left(\simeq 1 \mathrm{~km} \mathrm{~s}^{-1}\right.$ ) can lead to shattering. Moreover, even collisions with very small grains (projectile-to-target size ratio $\simeq 0.025$ ) will lead to complete disruption of a target for relative velocities of $100 \mathrm{~km} \mathrm{~s}^{-1}$. Hence, shattering is likely a very important destruction mechanism for large grains (Jones et al. 1994).

Impacting gaseous ions will sputter dust grains in strong shocks. We have reexamined the sputtering yields for astrophysically relevant materials. Extensive experimental and theoretical studies have led to the development of a “universal" sputtering relation (Bohdansky 1984). For any material, but independent of impacting ion, the relation for the sputtering yield contains one adjustable free parameter related to the penetration range of the impactors. The universal relation is fitted to recent experimental data to determine this free parameter, and very good fits are obtained for a variety of materials and impactor combinations. From an analysis of the extensive experimental data on sputtering of graphite, it is concluded that the sputtering process leads to the formation of an amorphous carbon surface layer. The binding energy of carbon atoms to this surface is only $4 \mathrm{eV}$ and hence, the sputtering yield near threshold is much larger than expected for the graphite binding energy $(7.5 \mathrm{eV})$. In contrast to previous studies (Seab \& Shull 1983), we conclude therefore that there is little difference in the sputtering of interstellar graphite and silicate grains near threshold.

The research of C. F. M. is supported in part through NSF grants AST 89-18573 and AST 92-21289. Theoretical studies of interstellar dust at NASA/Ames are supported through NASA grant 399-20-01-30 from the Astrophysics Theory Program.

\section{APPENDIX}

\section{IMPACT BLAST WAVES}

For a highly supersonic impact of a projectile on a target, the resulting blast wave is a self-similar flow of the second type (Barenblatt \& Zel'dovich 1972), in which the exponents in the solution, such as $\eta \equiv d \log R / d \log t$, are not determined by dimensional analysis. Physically, $\eta$ is determined by the rate at which energy flows out of the shocked cavity; mathematically, it is difficult to determine because the sonic surface is of unknown shape. If we express the decrease in energy as $E \propto R^{-k_{E}}$, then $\eta$ and $k_{E}$ are related by $\eta=2 /\left(5+k_{E}\right)$ (Ostriker \& McKee 1988). The numerical studies of Dienes $\&$ Walsh (1970) give $\alpha=0.59$ (see eq. [2.17]) for an impact blast wave in an ideal gas with $\gamma=1.5$; this corresponds to $\eta=0.371$ and $k_{E}=0.390$. For impacts in condensed materials at finite Mach number, they found $\alpha=0.58$, corresponding to $\eta=0.367$ and $k_{E}=0.45$, whereas for the lowest velocity impacts they found $\alpha \simeq 0.6\left(k_{E} \simeq \frac{1}{3}\right)$. We adopt $k_{E}=\frac{3}{8}$, corresponding to $\alpha=0.593$ and $\eta=0.372$, for the analysis of impact blast waves in grains in the text.

In order to gain some insight into the mechanism for energy loss from impact blast waves, we shall develop a simple model which incorporates its essential features. The outflow of the hot, shocked matter from the cavity may be described by two parameters: the mass-loss rate $\dot{M}$ and the exhaust velocity $v_{\text {ex }}$. Since the flow is self-similar, $\dot{M}$ is proportional to the mass flux across the shock front; hence, the exhaust mass fraction

$$
F \equiv \frac{\dot{M}}{2 \pi R^{2} \rho_{0} v_{s}}
$$

is constant, where we have assumed a hemispherical shape for the blast wave. The mass in the blast wave is then

$$
M=(1-F) \frac{2 \pi}{3} \rho_{0} R^{3},
$$

since it does not include the exhausted mass.

The momentum imparted to the blast wave by the exhaust is $\Psi=\frac{1}{2} M\langle v\rangle$, where $\langle v\rangle$ is the average velocity (relative to the target) of the material in the blast wave, and the factor $\frac{1}{2}$ allows for projection onto the symmetry axis. Adopting a linear velocity for the shocked matter $(v \propto r)$ and assuming a power-law density distribution (e.g., Ostriker \& McKee 1988), we obtain

$$
\langle v\rangle=\frac{3(\gamma+1)}{2(2 \gamma+1)} v_{1}=\frac{3}{2 \gamma+1} v_{s} .
$$

The rate at which the momentum increases is

$$
\dot{\Psi}=\dot{M} v_{\text {ex }} .
$$

For simplicity, we assume that the outflow is approximately one-dimensional, so that no projection factor enters here. If we define $k_{\Psi} \equiv-d \log \Psi / d \log R$ by analogy with $k_{E}$, we find

$$
k_{\Psi}=-\frac{2 F(2 \gamma+1)}{1-F}\left(\frac{v_{\mathrm{ex}}}{v_{s}}\right) .
$$


Next, consider the energy loss

$$
\dot{E}=-\frac{1}{2} \dot{M} v_{\mathrm{ex}}^{2} \text {. }
$$

Since the total energy is $E=\sigma M v_{s}^{2}$, where $\sigma$ is given by equation (2.19), we obtain

$$
k_{E}=\frac{3 F}{2(1-F) \sigma}\left(\frac{v_{\mathrm{ex}}}{v_{s}}\right)^{2} .
$$

To evaluate the energy loss, we introduce the basic assumption of our treatment, that the energy in the outflow is the same as the thermal energy content of the gas in a spherical Sedov-Taylor blast wave of the same energy:

$$
\frac{1}{2} v_{\mathrm{ex}}^{2} \equiv \frac{k T(F)}{(\gamma-1) \mu}
$$

where $T(F)$ is the temperature at the mass fraction $F$, and $\mu$ is the mean per particle. Anticipating that $k_{E}$ is small, we obtain $T(F)$ from Ostriker \& McKee's (1988) treatment of Kahn's approximation for energy-conserving blast waves; expansion of the equations about the origin gives

$$
\frac{T(F)}{T_{1}}=\left(\frac{\gamma+\delta}{\gamma+1}\right)^{\gamma-1} \exp \left[-\frac{3(\gamma-1)^{2}(1-\delta)}{\gamma(7-\gamma)}\right] F^{-1 / \gamma}
$$

where

$$
\delta=e^{1 / \gamma} F^{(7-\gamma) / 3 \gamma}
$$

is assumed small, and where $T_{1}=2\left[(\gamma-1) /(\gamma+1)^{2}\right] \mu v_{s}^{2} / k$ is the postshock temperature.

The system of equations for $k_{E}$ is closed by noting that dimensional analysis gives

$$
k_{E}=2 k_{\Psi}+3 \text {. }
$$

Solution of equations (A5), (A7)-(A11), and (2.19) gives $\left(\gamma, k_{E}\right)=(1.205,0.320),(1.5,0.372),(2,0.368)$. For $\gamma=7$, the linear velocity approximation is exact, and equation (A9) is replaced by $T(F) / T_{1}=F^{1 / 2}$, so that $k_{E}=0.268$. These values are approximately consistent with the results of Dienes $\&$ Walsh (1970) discussed above. In particular, they suggest that $k_{E}$ varies only slowly with $\gamma$. On the other hand, these results are inconsistent with the approximation discussed by Zel'dovich \& Raizer (1966), which gave $k_{E}=0.18$ at $\gamma=1.205$.

\section{REFERENCES}

Anders, E., Lewis, R., Tang, M., \& Zinner, E. 1989, in Interstellar Dust, ed. L. J. Allamandola \& A. G. G. M. Tielens (Dordrecht: Kluwer), 389

Andersen, H. H., \& Bay, H. L. 1981, in Sputtering by Particle Bombardment, ed. R. Behrisch (Berlin: Springer-Verlag), 145

Ahrens, T. J., \& O'Keefe, J. D. 1971, Moon, 4, 214

Allamandola, L. J., \& Tielens, A. G. G. M., ed. 1989, Interstellar Dust (Dordrecht: Kluwer)

Al'tshuler, L. V. 1965, Soviet Phys.-Uspekhi, 8, 52

Al'tshuler, L. V., Bakanova, A. A., Bushman, A. V., Dudoladov, I. P., \& Zubarev, V. N. 1977, Soviet Phys.-JETP, 46, 980

Asay, J. R., Trucano, T. G., \& Chhabildas, L. C. 1987, in Shock Waves in Condensed Matters, ed. S. C. Schmidt \& N. C. Holmes (Amsterdam: Elsevier), 159

Ashida, K., Ichimura, K., Matsuyama, M., \& Watanabe, K. 1984, J. Nucl. Mat., 128-129, 729

Ashida, K., Kanamori, K., Ichimura, K., Matsuyama, M., \& Watanabe, K. 1986, J. Nucl. Mat., 137, 288

Auciello, O., Haasz, A. A., \& Stangeby, P. C. 1985, Radiation Effects, 89, 63

Bach, H. 1970, Nucl. Instr. Meth., 84, 4

Bach, H., Kitzmann, I., \& Schroder, H. 1974, Radiation Effects, 21, 31

Barenblatt, G. I., \& Zel'dovich, Y. B. 1972, Ann. Rev. Fluid Mech., 3, 285

Barlow, M. J. 1978a, MNRAS, 183, 367

1978b MNRAS, 183, 397

Bar-nun, A., Herman, G., Rappaport, M. L., \& Mekler, Yu. 1985, in Ices in the Solar System, ed. J. Klinger (Dordrecht : Reidel), 287

Behrisch, R., ed. 1981, Sputtering by Particle Bombardment, I (Berlin: Springer-Verlag)

. 1983, Sputtering by Particle Bombardment, II (Berlin: SpringerVerlag)

Behrisch, R., Bohdansky, J., Oetjen, G. H., Roth, J., Schilling, G., \& Verbeek,

H. 1976, J. Nucl. Mat., 60, 321

Benson, S. W. 1976, Thermochemical Kinetics (New York: Wiley)

Betz, G., \& Wehner, G. K. 1983, in Sputtering by Particle Bombardment, II ed. R. Behrisch (Berlin: Springer-Verlag), 11

Bohdansky, J. 1980, J. Nucl. Mat., 93-94, 44

. 1984, J. Nucl. Instr. Meth. Phys. Res. B, 2, 587

Bohdansky, J., Bay, H. L., \& Ottenberg, W. 1978, J. Nucl. Mat., 76-77, 163

Bohdansky, J., \& Roth, J. 1985, Radiation Effects, 89, 49

Bohdansky, J., Roth, J., \& Bay, H. L. 1980, J. Appl. Phys., 51, 2861

Borders, J. A., Langley, R. A., \& Wilson, K. L. 1978, J. Nucl. Mat., 76, 168
Brown, W. L., Augustyniak, W. M., Brody, E., Cooper, B., Lanzerotti, L. J., Ramirez, A., \& Johnson, R. E. 1980, Nucl. Instr. Meth., 170, 321

Burenkov, A. Ph. 1986, Tables of the Parameters of Ion Implanted Species (New York: Gordon \& Breach)

Davis, J. W., \& Haasz, A. A. 1987, J. Nucl. Mat., 145-147, 349

Davis, J. W., Haasz, A. A., \& Stangeby, P. C. 1987, J. Nucl. Mat., 145-147, 417

De Carli, P.S., \& Jamieson, J. C. 1961, Science, 133, 1821

Dienes, J. K., \& Walsh, J. M. 1970, in High-Velocity Impact Phenomena, ed.

R. Kinslow (New York: Academic), 46

Draine, B. T. 1979, ApJ, 230, 106

Draine, B. T., \& Salpeter, E. E. 1979a, ApJ, 231, 77

1979b, ApJ, 231, 438

Edwin, R. P. 1973, J. Phys. D, 6, 833

Elman, B. S., Braunstein, G., Dresselhaus, M. S., Dresselhaus, G., Venkatesan, T., \& Gibson, J. M. 1984, Phys. Rev., B29, 4703

Gault, D. E. 1973, Moon, 6, 32

Gehrz, R. 1989, in Interstellar Dust, ed. L. J. Allamandola \& A. G. G. M. Tielens (Dordrecht: Kluwer), 445

Gould, R. K. 1975, J. Chem. Phys., 63, 1825

Greenberg, J. M. 1982, in Comets, ed. L. L. Wilkening (Tucson: Univ. of Arizona Press), 131

Hagen, W., Tielens, A. G. G. M., \& Greenberg, J. M. 1983, A\&A, 117, 132

Hechtl, E., Bohdansky, J., \& Roth, J. 1981, J. Nucl. Mat., 103-104, 333

Hollenbach, D., \& Salpeter, E. E. 1971, ApJ, 163, 155

Hornung, K., \& Mitchel, K. W. 1972, J. Chem. Phys., 56, 2072

Jenniskens, P., Baratta, G. A., Kouchi, A., de Groot, M. S., Greenberg, J. M., \& Strazulla, G. 1993, A\&A, 273, 583

Johnson, R. E., Barton, L. A., Boring, J. W., Jesser, W. A., Brown, W. L., \& Lanzerotti, L. J. 1985, in Ices in the Solar System, ed. J. Klinger (Dordrecht: Reidel), 301

Jones, A. P., \& Tielens, A. G. G. M. 1994, in The Cold Universe, ed Th. Montmerle (Gif-sur-Yvette: Editions Frontierès), in press

Jones, A. P., Tielens, A. G. G. M., Hollenbach, D. J., \& McKee, C. F. 1993, ApJ, submitted

1994, in preparation

Kahn, F. 1975, in Proc. 14th Internat. Cosmic Ray Conference (Munich), 11, 3566

Kelly, R. 1984, in Ion-Bombardment Modification of Surfaces, ed. O. Auciello \& R. Kelly (Amsterdam: Elsevier), 79

Kelly, R., \& Lam, N. Q. 1973, Radiation Effects, 19, 39 
Kelly, R., Lam, N. Q., Murti, D. K., Naguib, H. M., \& Parker, T. E. 1972, in Proc. Int. Summer School Physics of Ionized Gases, ed. M. Beograd (Split, Yugoslavia: Miljevac), 349

Kreyenhagen, K. H., \& Schuster, S. H. 1977, in Impact and Explosion Cratering, ed. D. J. Roddy, R. O. Pepin, \& R. B. Merrill (Elmsford, NY: Pergamon), 983

Lanzerotti, L. J., Brown, W. L., \& Johnson, R. E. 1985, in Ices in the Solar System, ed. J. Klinger (Dordrecht: Reidel), 317

Marsh, S. P. 1980, LASL Shock Hugoniot Data (Berkeley, CA: Univ. of California Press)

Mathis, J. S., Rumpl, W., \& Nordsieck, K. H. 1977, ApJ, 217, 425

Matsunami, N., et al. 1980, Radiation Effects Lett., 57, 15

McKee, C. F. 1989a, ApJ, 345, 782 $1989 \mathrm{~b}$, in Interstellar Dust, ed. L. J. Allamandola \& A. G. G. M. Tielens (Dordrecht: Kluwer), 431

McKee, C. F., \& Hollenbach, D. J. 1980, ARA\&A, 18, 219

McKee, C. F., Seab, C. G., Hollenbach, D. J., \& Tielens, A. G. G. M. 1987, ApJ, 318,674

McQueen, R. G., Marsh, S. P., Taylor, J. W., Fritz, J. N., \& Carter, W. J. 1970, in High-Velocity Impact Phenomenae, ed. R. Kinslow (New York: Academic), 294

Oeschner, H. 1985, in The Physics of Ionized Gases, ed. M. M. Popovic \& P. Krstic (Singapore: World Scientific), 571

O’Keefe, J. D., \& Ahrens, T. J. 1977, Proc. Lunar Sci. Conf., 8, 3357

Oort, J. H., \& van de Hulst, H. C. 1946, Bull. Astron. Soc. Netherlands, 10, 187

Ostriker, J., \& McKee, C. F. 1988, Rev. Mod. Phys., 60,

Pendleton, Y., Sandford, S. A., Allamandola, L. J., Tielens, A. G. G. M., \& Sellgren, K. 1994, in preparation

Phillipps, V., Flaskamp, K., \& Vietzke, E. 1982, J. Nucl. Mat., 111-112, 781

Philipps, V., \& Vietzke, E. 1987, in PAHs in Astrophysics, ed. A. Leger, L. d'Hendecourt, \& N. Bocarra (Dordrecht: Reidel), 95

Pillinger, C. T., Gardiner, L. R., \& Jull, A. J. T. 1976, Earth Planet. Sci. Lett., 33,289

Rae, W. J. 1970, in High Velocity Impact Phenomena, ed. R. Kinslow (New York: Academic) 214

Rao, M. P. R. 1985, in Shock Waves in Condensed Matter, ed. Y. M. Gupta (New York: Plenum), 681

Rocard, F., Benit, J., Bibring, J. P., Ledu, D., \& Meunier, R. 1986, Radiation Effects, 99,97

Rosenberg, D., \& Wehner, G. K. 1962, J. Appl. Phys., 33, 1842

Roth, J. 1983 in Sputtering by Particle Bombardment, II (Berlin: SpringerVerlag), 91

Roth, J., Bohdansky, J., \& Ottenberger, W. 1979, Rpt. Ipp. 9/26 (MPI Plasmaphysik, Garching)
Roth, J., Bohdansky, J., Poschenreider, W., \& Sinha, M. K. 1976, J. Nucl. Mat., 63,222

Roth, J., Bohdansky, J., \& Wilson, K. L. 1982, J. Nucl. Mat., 111-112, 775

Seab, C. G. 1987, in Interstellar Processes, ed. D. J. Hollenbach \& H. Thronson (Dordrecht: Reidel), 491

Seab, C. G., \& Shull, J. M. 1983, ApJ, 275, 652

Sedov, L. I. 1959, Similarity and Dimensional Methods in Mechanics (New York: Academic)

Shull, J. M. 1977, ApJ, 215, 805

. 1987, ApJ, 226, 858

Sigmund, P. 1981, in Sputtering by Particle Bombardment, I, ed. R. Behrisch (Berlin: Springer-Verlag), 9

Smith, J. N., Meyer, C. H., \& Layton, J. K. 1976, Nucl. Tech., 29, 318 1977, J. Nucl. Mat., 67, 234

Strazulla, G., Baratta, G. A., \& Magazzu, A. 1985, Astron. Express, 1, 143

Strazulla, G. L., Calcagno, L., \& Foti, G. 1985, Nuovo Cimento, 8, 63

Tielens, A. G. G. M. 1989, in IAU Symp. 135, Interstellar Dust, ed. L. J. Allamandola \& A. G. G. M. Tielens (New York: Reidel), 239

. 1990, in Carbon in the Galaxy, ed. J. C. Tarter, S. Chang, \& D. C deFrees (NASA SP-3061), 59

Tielens, A. G. G. M., \& Allamandola, L. J. 1987, in Interstellar Processes, ed. D. J. Hollenbach \& H. A. Thronson (New York: Reidel), 397

Tielens, A. G. G. M., \& Hagen, W. 1982, A\&A, 224, 245

Tielens, A. G. G. M., \& Hollenbach, D. J. 1985, ApJ, 291, 722

Tielens, A. G. G. M., Seab, C. G., Hollenbach, D. J., \& McKee, C. F. 1987, ApJ, 319, L109

Venkatesan, T., Dynes, R. C., Wilkens, B., White, A. E., \& Hamm, R. 1984, Nucl Instr. Meth. Phys. Res. B, 1, 599

Vietzke, E., Flaskamp, K., \& Philipps, V. 1982, J. Nucl. Mat., 111-112, 763

. 1984, J. Nucl. Mat., 128-129, 545

von Seefeld, H., Schmidl, H., Behrisch, R., \& Scherzer, B. M. U. 1976, J. Nucl. Mat., 63, 215

Watson, W. D. 1973, in Interstellar Dust, ed. J. M. Greenberg (Dordrecht: Reidel), 335

Wright, R. B., Varma, R., \& Gruen, D. M. 1976, J. Nucl. Mat., 63, 415

Yamada, R. 1987, J. Nucl. Mat., 145-147, 359

Yamada, R., Nakamura, K., Sone, K., \& Saidoh, M. 1980, J. Nucl. Mat., 95, 278

Yamamura, Y., Matsunami, N., \& Itoh, N. 1983, Radiation Effects, 71, 65

Yin, L. I., Ghose, S., \& Adler, I. 1972, J. Geophys. Res., 77, 1360

Zel'dovich, Y. B., \& Raizer, Y. P. 1966, Physics of Shock Waves and High Temperature Phenomenae (New York: Academic) 